\title{
Modeling and $H_{\infty}$ Control of Active-Passive Vibration Isolation for Floating Raft System
}

\author{
Beibei Yang, Yefa Hu, and Jinguang Zhang \\ School of Mechanical and Electronic Engineering, Wuhan University of Technology, Wuhan 430070, China \\ Correspondence should be addressed to Beibei Yang; wallace1987@whut.edu.cn
}

Received 21 November 2016; Revised 16 January 2017; Accepted 23 January 2017; Published 21 February 2017

Academic Editor: Orest V. Iftime

Copyright (C) 2017 Beibei Yang et al. This is an open access article distributed under the Creative Commons Attribution License, which permits unrestricted use, distribution, and reproduction in any medium, provided the original work is properly cited.

\begin{abstract}
This paper presents a new approach for constructing a mathematical model of the floating raft system directly from input-output measurements in the presence of noise. In contrast to the original OKID/ERA algorithm, which works through the observer Markov parameters, the new approach used observer output residuals to convert the initial stochastic identification to a virtually deterministic identification problem. The extension of deterministic algorithm to stochastic problems by proposed stochasticto-deterministic conversion can be done with ease. A MIMO (multiple-input multiple-output) system state-space model and an associated Kalman filter gain can be identified. $H_{\infty}$ controller with high robustness to model error is designed to solve multifrequency varying vibration for floating raft system. Both simulated and experimental results confirm the validity and the benefits of the approach.
\end{abstract}

\section{Introduction}

A floating raft system is a special double-layer isolation system aiming to reduce the level of noise and vibration and has been widely applied to many kinds of ships and submarines $[1,2]$. It can isolate vibration of hosts and auxiliary machines and reduce the structural noise of ships and submarines effectively. It can also protect equipment and instruments in ships and submarines from being damaged and makes them operate properly when ships and submarines are subjected to external loads and sudden shocks [3]. However, in real applications, two and more machines are mounted on the floating raft system and their working frequencies are usually multifrequency and time varying [4]. The floating raft system includes vibrations of the flexible floating raft and of the rigid motors. Therefore, the floating raft system is a complex system generally subject to multifrequency varying vibration.

Theoretical modeling for floating raft systems can be summarized as multibody dynamics [5], impedance mode method [6], four-terminal parameter method [7], finite element method [8], statistical energy analysis [9], power flow method [10], and so on. The interactions between active actuators and active actuators on the floating raft system are ignored by these approaches. Meanwhile, the models are established on many assumptions that do not always hold.

In response to these obstacles, we take a system identification approach, constructing a mathematical model of the dynamic system directly from measured input-output data. The identified model can then be used to predict the response of the system to any excitation. In real applications, noise inevitably affects the measured data, making the identification problem stochastic. In the presence of noise, it would be desirable to identify from the experimental data not only the model of the system, but also the noise characteristics [11]. This presumes that the same sensors and actuators used in the identification tests will also be employed in the control system designed around the identified model. One of the most successful identification algorithms for linear state-space models is OKID/ERA (observer/Kalman filter identification) [12], which relies on an observer equation to compress the dynamics of the system and efficiently estimate its Markov parameters. The latter are then passed to the Eigensystem Realization Algorithm (ERA) [13] or some improved variants of it, for example, ERA with Data Correlation (ERA/DC) [14], to complete the identification process. The observer at the core of the method was proven to be the steady-state Kalman 
filter corresponding to the system to be identified and to the covariance of the process and measurement noise. A remarkable result of OKID/ERA is that the method provides simultaneously both the system matrices and the Kalman gain, extracting all the possible information present in the data.

OKID/ERA has been successfully applied for over twenty years, especially in the aerospace community (it was originally distributed by NASA for the identification of lightly damped structures), and it keeps receiving attention as researchers try to further improve it $[15,16]$ and apply it to linear time-varying problems [17] or even to nonlinear systems [18]. Additionally, OKID has been recently extended to the case where only output time histories are measured, leading to output-only observer/Kalman filter identification (O3KID) [19]. In this paper we show how ERA (or ERA/DC) is not the only method to complete the identification process. Thanks to a novel interpretation of the main OKID result, we prove that it is possible to use a Kalman filter to optimally transform a problem of identification from noisy data into a simpler, noise-free problem. As a result, we propose and demonstrate with examples several new OKID-based identification algorithms optimal in the presence of noise, which can be as many as the number of deterministic identification algorithms that one can find. We establish then the Kalman filter as the bridge from stochastic-to-deterministic system identification and OKID as a unified optimal approach to handle noisy data in system identification, paralleling the central role that the Kalman filter has in signal estimation.

In active noise control (ANC) for floating raft system [20], the filtered-x least mean square (FXLMS) algorithm [21] is the most popular adaptive feedforward algorithm to update the controller, as it enjoys good applicability for real-life applications in terms of both noise reduction performance and implementation cost. Some variants of the FXLMS algorithm [22-24] have been studied to reduce computational complexity or to improve convergence rate and the control of impulsive noise. As one of feedforward algorithms, FXLMS control algorithm has a common assumption that reference signal is stable periodic signal by default. However, in real applications, two and more machines are mounted on the floating raft system and their working frequencies are usually multifrequency and frequency varying.

In response to these obstacles, feedback control algorithms are mainly applied in structural vibration control and suppressing multifrequency varying vibration. LPV (Linear Parameter Varying) technology is used to update controller parameters according to the estimated frequency in real-time for suppressing the large range frequency varying vibration [25]. For the narrowband disturbance rejection problem of helicopter tail, LQR (Linear Quadratic Regulator) control algorithm and $H_{\infty}$ control algorithm can suppress frequency varying vibration in a small range, and experiment shows that $H_{\infty}$ control algorithm has the better performance than LQR control algorithm [26]. Adaptive LQ control algorithm with frequency estimator is applied to eliminate frequency varying disturbances of the diesel engine. Then, 3-input/3-output active vibration control experiments were conducted in the floating raft system [27]. The difference between identified model and system model can be described by using additive uncertainty and multiplicative uncertainty in $H_{\infty}$ control algorithm [28]. $H_{\infty}$ controller is based on high robustness, which can effectively solve the problem of overflow instability. Meanwhile, frequency response function of the closed-loop system can be reshaped by the design method based on the mixed sensitivity problem for $H_{\infty}$ control algorithm [29]. Thus, the multifrequency varying vibration problem can be effectively solved.

The paper is organized as follows. After rigorously formulating the stochastic system identification problem, the OKID core equation is derived, in a slightly different way with respect to [30] in order to better highlight the central role of the Kalman filter in system identification. Then the novel interpretation of the main OKID result is presented and it is shown how to convert the original stochastic problem into an equivalent deterministic form. The resulting new algorithms are outlined and their features are illustrated via a simple numerical example, which provides the ground to present the conceptual contribution of the work. An experimental example on a floating raft platform is given to show the method in action on a more realistic system. The MIMO system model can be identified and the order of identified model can be reduced by retaining pulse response weighting indices of every state element to the overall dynamics. $H_{\infty}$ controller can be designed by mixed sensitivity problem according to the reduced model identified by OKID. The identified model from input-output data and $H_{\infty}$ controller are demonstrated via both numerical and experimental examples on floating raft platform with varying frequency vibration source.

\section{Problem Description}

Figure 1 shows that the analytical model of the floating raft system includes using springs and electromagnetic actuators. The overall system can be divided into several subsystems: vibration source $\left(M_{A}\right.$ and $\left.M_{B}\right)$, floating $\operatorname{raft}\left(M_{R}\right)$, foundation $\left(M_{b}\right)$, and isolators $(U, T$ and $D)$. According to [31], the mathematical model of floating raft system with the dynamic equations of substructures can be expressed as

$$
M \ddot{X}+K X=F(t),
$$

where $X \in \mathbb{R}^{(18+N) \times 1}$ represents coordinate matrix to describe the motion of the floating raft system and $M \epsilon$ $\mathbb{R}^{(18+N) \times(18+N)}$ is total mass matrix of floating raft system. $K \in \mathbb{R}^{(18+N) \times(18+N)}$ is the modal stiffness matrix of the system. $F(t) \in \mathbb{R}^{(18+N) \times(18+N)}$ is the load matrix of system.

$$
\begin{gathered}
X=\left[x_{A}, y_{A}, z_{A}, \theta_{A}, \phi_{A}, \varphi_{A}, x_{B}, y_{B}, z_{B}, \theta_{B}, \phi_{B}, \varphi_{B}, x_{R}, y_{R},\right. \\
\left.z_{R}, \theta_{R}, \phi_{R}, \varphi_{R}, q_{1}, q_{2}, \ldots, q_{N}\right]^{T}, \\
M=\operatorname{diag}\left\{M_{A}, M_{A}, M_{A}, J_{A x}, J_{A y}, J_{A z}, M_{B}, M_{B}, M_{B}, J_{B x},\right. \\
\left.\quad J_{B y}, J_{B z}, M_{R}, M_{R}, M_{R}, J_{R x}, J_{R y}, J_{R z}, 1,1,1, \ldots, 1\right\}, \\
F(t)=\left[F_{A x}(t), F_{A y}(t), F_{A z}(t), M_{A \theta}(t), M_{A \phi}(t),\right. \\
M_{A \varphi}(t), F_{B x}(t), F_{B y}(t), F_{B z}(t), M_{B \theta}(t), M_{B \phi}(t), \\
M_{B \varphi}(t), F_{R x}(t), F_{R y}(t), F_{R z}(t), M_{R \theta}(t), M_{R \phi}(t), \\
\left.M_{R \varphi}(t), 0,0, \ldots, 0\right],
\end{gathered}
$$




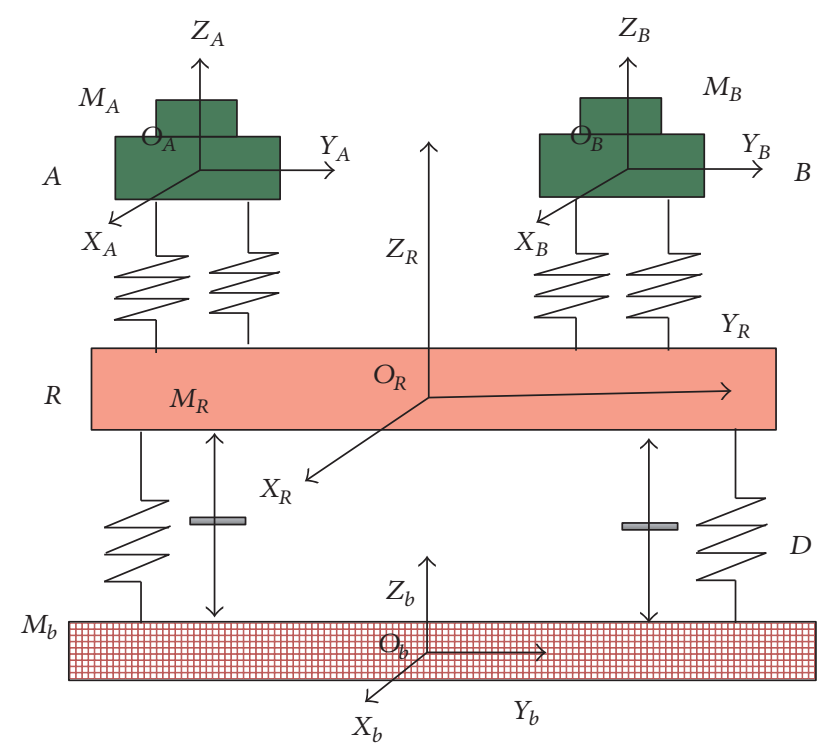

FIGURE 1: Analytical model of floating raft system.

where $x_{A}, y_{A}, z_{A}, x_{B}, y_{B}, z_{B}, x_{R}, y_{R}$, and $z_{R}$ are, respectively, translation displacements of mass center of $A, B$, and $R$. $\theta_{A}, \phi_{A}, \varphi_{A}, \theta_{B}, \phi_{B}, \varphi_{B}, \theta_{R}, \phi_{R}$, and $\varphi_{R}$ are, respectively, attitude coordinate system of $A, B$, and $R$. In order to describe the elastic vibration of the floating raft, the former $N$ order mode $q_{1}, q_{2}, \ldots, q_{N}$ is retained. $M_{A}, M_{B}$, and $M_{R}$ are, respectively, mass of subsystems $A, B$, and $R, J_{A x}, J_{A y}$, $J_{A z}, J_{B x}, J_{B y}, J_{B z}, J_{R x}, J_{R y}$, and $J_{R z}$ are, respectively, rotational inertia of objects $A, B$, and $R$ around the $x$-, $y$ - and $z$-axes. $F_{A x}(t), F_{A x}(t), F_{A x}(t), M_{A \theta}(t), M_{A \phi}(t), M_{A \varphi}(t), F_{B x}(t), F_{B x}(t)$, $F_{B x}(t), M_{B \theta}(t), M_{B \phi}(t), M_{B \varphi}(t), F_{R x}(t), F_{R x}(t), F_{R x}(t), M_{R \theta}(t)$, $M_{R \phi}(t)$, and $M_{R \varphi}(t)$ are, respectively, external disturbance forces of subsystems $A, B$, and $R$.

In the active vibration isolation system, the structure diagram of electromagnetic actuator is shown in Figure 2. The electromagnetic actuator consists of the following components: (1) mandrel, (2) flexible spring, (3) yoke iron, (4) permanent, (5) coil, and (6) linear bearing. The electromagnetic force generated by the energized coil is passed from the armature iron to the floating raft. The mandrel of electromagnetic actuator is connected with the floating raft. The accelerometer is placed on the floating raft near the electromagnetic actuator to measure the error signal. The electromagnetic force $F_{e}$ generated by the electromagnetic actuator is expressed as follows:

$$
F_{e}=N B I L
$$

where $N$ is number of coil turns, $B$ is the average magnetic induction of the air gap, $I$ is the current value of the coil, and $L$ is the effective length of the coil for cutting magnetic field lines.

According to the dynamic equation (1), the model of floating raft system is a linear system and a state-space equation can be established. However, the identification of the complete set of coefficients which appear in (1), (2a), (2b), (2c), and (3) is a rather complex task for the very high number of parameters, little nonlinearities, and space-time variant effects. The decoupled models are expressed in [31], under the assumption of small deviations of rigid motions to generate a set of linear time-invariant models. On the other hand, a better description of floating raft dynamics can be obtained in terms of MIMO coupled models, determined on the basis of physical laws or by system identification techniques.

We take the approach of finding a mathematical model of the floating raft by system identification. A dynamic system can be represented with different types of model. Statespace models are very popular in many applications, mainly because they lend themselves to analysis via well-established analytical and numerical tools from linear algebra. Consider the following linear dynamical system in discrete-time statespace form:

$$
\begin{aligned}
x(k+1) & =A x(k)+B u(k)+w_{p}(k), \\
y(k) & =C x(k)+D u(k)+w_{m}(k),
\end{aligned}
$$

where $x \in \mathbb{R}^{n \times 1}$ is the state vector, $u \in \mathbb{R}^{m \times 1}$ is input vector, $y \in \mathbb{R}^{q \times 1}$ is the output vector, $A \in \mathbb{R}^{n \times n}$ is the system matrix, $B \in \mathbb{R}^{n \times m}$ is the input matrix, $C \in \mathbb{R}^{q \times n}$ is the output matrix, and $D \in \mathbb{R}^{q \times m}$ is the direct influence matrix. $w_{p} \in \mathbb{R}^{n \times 1}$ is the process noise assumed to be Gaussian, zero mean, and white with the covariance matrix $Q \in \mathbb{R}^{n \times n}$, and $w_{m} \in \mathbb{R}^{q \times 1}$ is the measurement noise with the same assumption as $w_{p}$ but a different covariance matrix $R \in \mathbb{R}^{q \times q}$. The sequences $w_{p}$ and $w_{m}$ are assumed to be statistically independent of each other. The system identification problem can be stated as follows. Given a set of input-output data, measured from the system starting at some unknown initial state $x(0)$,

$$
\begin{aligned}
& \{u(k)\}=\{u(0), u(1), u(2), \ldots, u(l-1)\}, \\
& \{y(k)\}=\{y(0), y(1), y(2), \ldots, y(l-1)\} .
\end{aligned}
$$

The objective is to identify the system in (1), that is, to find the matrices $A, B, C$, and $D$. The data is assumed to be of sufficient length and richness so that the system of (4a) and (4b) can be correctly identified. Neither the noise sequences $\left\{w_{p}(k)\right\}$ and $\left\{w_{m}(k)\right\}$ or their covariance matrices $R$ and $Q$ are known. As mentioned in the introduction, it would be ideal to extract from the measured input-output data also the optimal linear observer of the system state, that is, the Kalman gain $K$. It is demonstrated later in the paper how not only it is desirable to estimate $K$, but the identification of the system via the identification of the optimal observer is superior to the direct identification of the system. In other words, the estimation of $K$ can be seen as a valuable by-product of the proposed system identification approach.

\section{New Approach to System Identification}

The new identification strategy consists in two main parts. As in OKID/ERA, we start from an observer equation to derive an expression between the input and the output without the state appearing explicitly. This results in the OKID core equation, whose least-squares (LS) solution establishes that the observer used in the derivation is the optimal observer 

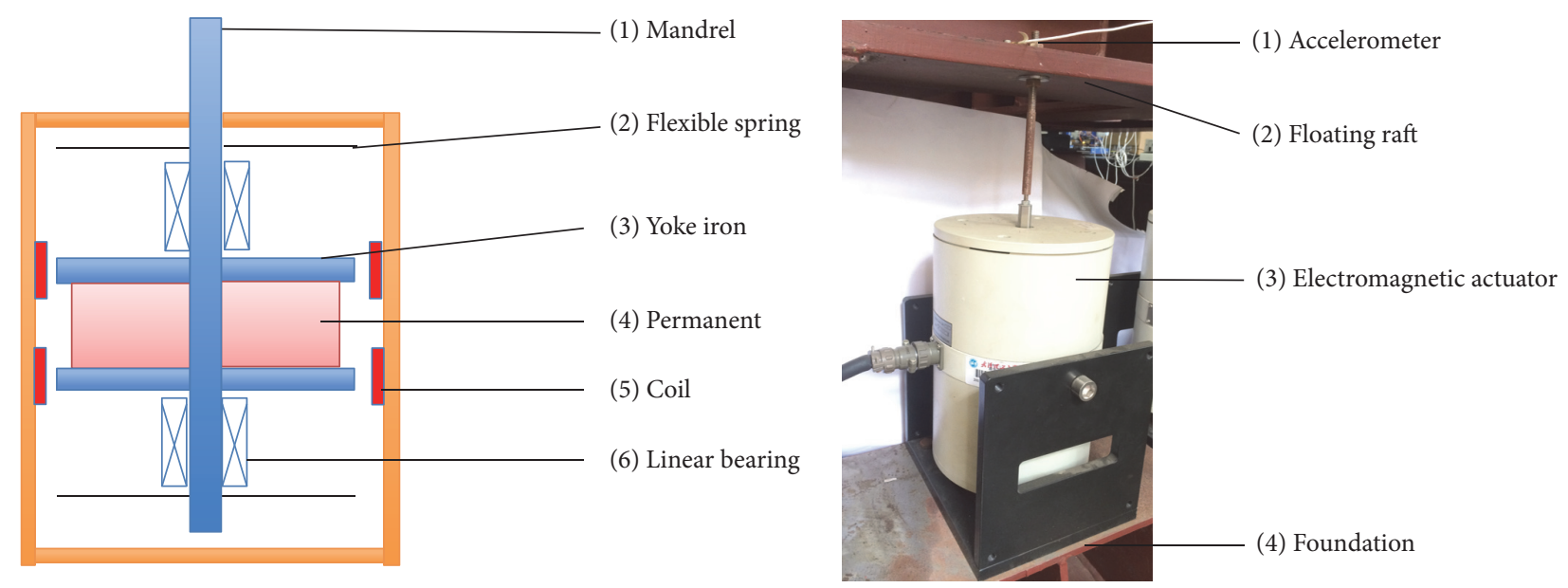

FIGURE 2: The structure diagram of electromagnetic actuator.

in the presence of noise (Kalman filter). In contrast to OKID/ERA, the OKID core equation is used to estimate the Kalman filter output residuals. In the second part, we use the estimated residuals to construct a new identification problem with nominally no noise in its formulation. The only source of noise is the estimation error in the observer residuals. The dynamic system to be identified in the new problem is the Kalman filter. From the matrices of the Kalman filter, those of the system can be easily recovered.

The overall identification process is then conceptually more direct than OKID/ERA, where the identification observer Markov parameters had to be converted into the system Markov parameters before applying ERA. References $[32,33]$ devised a subspace technique for linear system identification based on the preliminary estimation of the Kalman filter output residuals. Their purpose was to develop a subspace method applicable to closed-loop identification, getting around the correlation between future input and past residuals that characterizes closed-loop identification problems. The novelty of this paper lies in using the estimated residuals to convert a stochastic system identification problem into a deterministic system identification problem to be solved with existing and well-established methods. Regarding the estimation of the Kalman filter output residuals, [11] is also worth mentioning, where estimates of the residuals were used to design the Kalman filter itself. However, in [11] knowledge of the system was assumed. In the present work we estimate output residuals to identify both the system and the associated Kalman filter.

3.1. Estimation of Kalman Filter Output Residuals. Consider the following observer for the system in (4a) and (4b):

$$
\begin{aligned}
\widehat{x}(k+1) & =A \widehat{x}(k)+B u(k)+K(y(k)-\widehat{y}(k)), \\
\widehat{y}(k) & =C \widehat{x}(k)+D u(k),
\end{aligned}
$$

where $\hat{x} \in \mathbb{R}^{n \times 1}$ and $\hat{y} \in \mathbb{R}^{q \times 1}$ are the observer state and output and $K \in \mathbb{R}^{n \times q}$ is the observer gain. The observer's role is to estimate the actual system state $x$ from the past inputoutput measurements. It is then a state estimator or, in the control engineering terminology, a state observer. Because all the matrices in (6a) and (6b) are constant with time, the observer is a linear time-invariant (LTI) model. Defining the observer output residuals as

$$
\varepsilon(k)=y(k)-\widehat{y}(k)
$$

and plugging (6b) into (6a) and (7) into (6b), the observer in (6a) and (6b) can be written in the equivalent form

$$
\begin{aligned}
\widehat{x}(k+1) & =\bar{A} \hat{x}(k)+\bar{B} v_{x}(k), \\
y(k) & =C \widehat{x}(k)+D u(k)+\varepsilon(k),
\end{aligned}
$$

where $\bar{A}=A-K C, \bar{B}=\left[\begin{array}{ll}B-K D & K\end{array}\right]$, and $v_{x}(k)=\left[\begin{array}{l}u(k) \\ y(k)\end{array}\right]$. Equations (8a) and (8b) are referred to as the predictor form of the observer in (6a) and (6b). Propagating (8a) forward in time by $p$ time steps and then shifting the time index backward by $p+1$, we obtain

$$
\widehat{x}(k)=\bar{A}^{p} \hat{x}(k-p)+T z(k),
$$

where

$$
\begin{aligned}
& T=\left[\begin{array}{lllll}
\bar{B} & \overline{A B} & \ldots & \bar{A}^{p-2} \bar{B} & \bar{A}^{p-1} \bar{B}
\end{array}\right], \\
& z(k)=\left[\begin{array}{c}
v_{x}(k-1) \\
v_{x}(k-2) \\
\vdots \\
v_{x}(k-p)
\end{array}\right] .
\end{aligned}
$$

As proven later, the observer in (9) is stable. Such a property guarantees that $\bar{A}^{p}$ becomes negligible for sufficiently large values of $p(p \gg n)$. Equation (9) yields then the following relation expressing the current state as a function of past input and output values:

$$
\widehat{x}(k)=T z(k) .
$$


Plugging (11) into (8b), we obtain the classic OKID equation:

$$
y(k)=\Phi v(k)+\varepsilon(k)
$$

where $\Phi$ and $v(k)$ are augmented version of $T$ and $z(k)$ to account for the direct influence of input on the output through the $D$ matrix,

$$
\begin{aligned}
& \Phi=\left[\begin{array}{llllll}
D & C \bar{B} & C \overline{A B} & \cdots & C \bar{A}^{p-1} \bar{B}
\end{array}\right], \\
& v(k)=\left[\begin{array}{c}
u(k) \\
v_{x}(k-1) \\
v_{x}(k-2) \\
\vdots \\
v_{x}(k-p)
\end{array}\right] .
\end{aligned}
$$

Equation (9) relates measured input and output, without the state appearing explicitly. In the time-series literature it is known as an autoregressive model with exogenous input (ARX). Also, note that $\Phi \in \mathbb{R}^{q \times q p}$ contains the sequence of Markov parameters (or unit pulse response) of the observer in predictor form. Equation (12) can be written for each time step $k=p, p+1, \ldots, l-1$ of the measured data record, to obtain the following set of equations in matrix form:

$$
Y=\Phi V+E
$$

where

$$
\begin{aligned}
Y & =\left[\begin{array}{llll}
y(p) & y(p+1) & \cdots & y(l-1)
\end{array}\right], \\
V & =\left[\begin{array}{llll}
v(p) & v(p+1) & \cdots & v(l-1)
\end{array}\right], \\
E & =\left[\begin{array}{llll}
\varepsilon(p) & \varepsilon(p+1) & \cdots & \varepsilon(l-1)
\end{array}\right] .
\end{aligned}
$$

Equation (14) is at the core of OKID. $Y$ and $V$ are known (from measurements), and $\Phi$ and $E$ are not. By having a large number of data (large $l$ ) and considering $E$ as an error term, it is possible to find the LS solution to (14)

$$
\widetilde{\Phi}=Y V^{T}\left(V V^{T}\right)^{-1}=Y V^{\dagger},
$$

where $\dagger$ denotes the Moore-Penrose pseudoinverse of a matrix, right-multiplying (14) by $V^{T}$ and replacing $\Phi$ with $\widetilde{\Phi}$, we obtain

$$
Y V^{T}=Y V^{T}\left(V V^{T}\right)^{-1} V V^{T}+E V^{T}=Y V^{T}+E V^{T}
$$

which implies that $E V^{T}=0$. Since the stated assumptions make the process of (4a) and (4b) stationary, then for large $l$ it can be proven that the residuals $\varepsilon$ of LS problem of (14) are then orthogonal to the current and past input values and to the past output values. This is the same property that uniquely characterizes the Kalman filter output residuals, which proves that the solution to the LS problem of (14) provides an estimate of the output residuals of Kalman filter associated with unknown system matrices $A, B, C$, and $D$ and noise statistics $Q, R$ that generated the input-output data in (5a) and (5b). Among all the possible observers, the Kalman filter is optimal in the sense that it minimizes the expected 0 value of the squared norm of the state estimation error $E\left[(x(k)-\widehat{x}(k))^{T}(x(k)-\widehat{x}(k))\right]$ at each time step $k$. Under the assumption of stationary noise (constant $Q$ and $R$ ) and after a certain number of steps $p$ after which the filter transient has vanished, the optimal gain becomes constant in time and is referred to as steady-state Kalman gain. Given the system matrices $A, B, C$, and $D$ and noise statistics $Q, R$, the steady-state Kalman gain $K$ can be computed from the wellknown algebraic equation. The choice of the letter $K$, usually reserved for the Kalman gain, for the gain of the observer in (6a) and (6b) is now justified. As a corollary, $\widetilde{\Phi}$ contains the estimates of the Markov parameters of the Kalman filter. The original OKID/ERA algorithm would compute the Markov parameters of the system from the ones of Kalman filter and feeding them to ERA (or ERA/DC) would find a realization of the matrices $A, B, C, D$, and $K$ as desired. Instead, in the new approach presented in this paper, we focus on the output residuals of the Kalman filter. Thanks to the above proof, their sequence can be estimated from (14)

$$
\widetilde{E}=Y-\widetilde{\Phi} V
$$

For simplicity of notation, the estimated residuals will be denoted in the rest of the paper simply as $\varepsilon(k)$, with no tilde.

3.2. Identification of the Observer/Kalman Filter. Recalling (7), (6a) and (6b) can be written as follows:

$$
\begin{aligned}
\widehat{x}(k+1) & =A \hat{x}(k)+B u(k)+K \epsilon(k), \\
\hat{y}(k) & =C \hat{x}(k)+D u(k)
\end{aligned}
$$

which is usually known in the literature as the innovation form of the Kalman filter. Equations (19a) and (19b) can also be looked at as the state-space model of a dynamic system with $u$ and $\epsilon$ as input and $\widehat{y}$ as output. Such interpretation is at the basis of the work presented in this paper. Indeed, once an estimate for the time history of the Kalman filter residual $\epsilon$ is available, that can be used to obtain via (7) an estimate for the time history of the Kalman filter output $\hat{y}$ as well. Both the input and the output sequences of the dynamic system in (19a) and (19b) are then known. Additionally, in (19a) and (19b) no (unknown) noise term is present. We have just constructed a new noise-free identification problem: given the time histories of $u, \epsilon$, and $\widehat{y}$, find the matrices $A, B, C$, $D$, and $K$. Thanks to the absence of noise, any deterministic identification method can be used to solve the new problem. Note that the solution to the new problem is also the solution to the original problem.

This gives rise to many OKID-based identification algorithms, as many as the deterministic identification methods one can think of. In this paper, to illustrate the effectiveness of the new approach, we demonstrate via examples two possible choices, namely, the deterministic intersection (DI) and the deterministic projection (DP) method. The DI and DP methods are considered deterministic because their formulation is based on purely deterministic state-space models (with no process or measurement noise). The resulting new OKIDbased algorithms are referred to as OKID/DI and OKID/DP 
to remark that the underlying Kalman filter is identified in its innovation form, distinguishing them from the following variant.

An alternative way to complete the identification is given by an equivalent state-space model to describe the dynamics of the Kalman filter. Recalling (7), (8a) and (8b) can be rewritten as

$$
\begin{aligned}
\widehat{x}(k+1) & =\bar{A} \widehat{x}(k)+\bar{B} v_{x}(k), \\
\widehat{y}(k) & =C \widehat{x}(k)+D u(k) .
\end{aligned}
$$

Similar to the innovation form, (20a) and (20b) represent a dynamic system whose input $u$ and $y$ and output $\hat{y}$ are known. Any deterministic identification method can be applied to find a realization of the matrices $\bar{A}, \bar{B}, C$, and $D$, from which $K, B$, and $A$ can be recovered to complete the identification of the system in (4a) and (4b). This alternative is illustrated in [34], where numerical examples showed that it is less robust than the algorithms based on the Kalman filter in innovation form. Therefore, the algorithms based on (20a) and (20b) are not illustrated in this paper.

It is worth adding that other algorithms based on the new identification strategy can be devised simply by replacing DI and DP by other deterministic methods. As a last comment, it is noted that the input to the observer to be identified is different in the state and observation equations. More precisely, the state equation has an additional input $\epsilon$, which makes the form of the deterministic identification problem slightly different from the standard form usually considered in the literature, including the DI and DP algorithms. Two ways to address the issue are possible. One consists in feeding the deterministic identification algorithms with the same additional input in the observation equation as well, relying on the associated coefficients in the corresponding extended $D$ matrix being identified as 0 . The other approach is to tailor the deterministic identification algorithms so that they identify the observer taking into account its peculiar form. The required modification is very simple to apply, for example, in the case of the deterministic intersection methods.

3.3. Algorithm. The detailed steps to implement the new method are given below. The inputs to the algorithm are the sequences $\{u(k)\}$ and $\{y(k)\}$ from (5a) and (5b). The output is the set of identified matrices $A, B, C, D$, and $K$.

(1) Construct the matrices $Y$ and $V$ from (15a) and (15b).

(2) Compute

$$
\begin{aligned}
\widetilde{\Phi} & =Y V^{\dagger}, \\
{\left[\begin{array}{llll}
\hat{y}(p) & \widehat{y}(p+1) & \cdots & \widehat{y}(l-1)
\end{array}\right] } & =\widetilde{\Phi} V .
\end{aligned}
$$

(3) Compute

$$
[\epsilon(p) \epsilon(p+1) \cdots \epsilon(l-1)]=Y-\widetilde{\Phi} V
$$

(4) Define the following input and output sequences:

$$
\begin{aligned}
& \left\{u_{\text {det }}\right\}=\left\{\left[\begin{array}{l}
u(p) \\
\epsilon(p)
\end{array}\right]\left[\begin{array}{l}
u(p+1) \\
\epsilon(p+1)
\end{array}\right] \cdots\left[\begin{array}{l}
u(l-1) \\
\epsilon(l-1)
\end{array}\right]\right\}, \\
& \left\{y_{\text {det }}\right\}=\{\hat{y}(p) \hat{y}(p+1) \cdots \hat{y}(l-1)\} .
\end{aligned}
$$

(5) Execute, with input $\left\{u_{\text {det }}\right\}$ and output $\left\{y_{\text {det }}\right\}$, and read the output matrices $A_{\mathrm{det}}, B_{\mathrm{det}}, C_{\mathrm{det}}$, and $D_{\mathrm{det}}$.

(i) The DP algorithm for OKID/DP

(ii) The DI algorithm for OKID/DI

(iii) Any other algorithm for deterministic linear state-space model identification from arbitrary excitation

(6) Extract the desired matrices

$$
\begin{aligned}
& A=A_{\text {det }}, \\
& B=B_{\text {det }}(:, 1: m), \\
& C=C_{\text {det }}, \\
& D=D_{\text {det }}(:, 1: m), \\
& K=B_{\text {det }}(:, m+1: m+q) .
\end{aligned}
$$

3.4. Example. To demonstrate the identification procedure we generate input-output data as follows. Figure 3 presents structure diagram of floating raft experimental tests for input-output data. The input excitation $\{u(k)\}$ is provided in correspondence of two electromagnetic actuators as a vertical force. The excitation is obtained via an electrical command to two actuators in the form of a band-limited $(0-200 \mathrm{~Hz})$ white process normally distributed with zero mean and standard deviation of $0.1 \mathrm{~V}$. With regard to the structural response $\{y(k)\}$, two vertical acceleration signals on the top of floating raft around the installation point of electromagnetic actuators are measured. The analog sensor signals are discretized with a sampling frequency of $2560 \mathrm{~Hz}$. The resulting time histories of $12.8 \mathrm{~s}$ in duration contain $32768 \times 2$ time samples to be used for the identification of the state-space model.

Testing the identified models on an independent set of data is a meaningful way to compare different models, since the exact true values of real structure modal parameters are not known. The same 4 stochastic system identification methods are used to identify the system and the associated Kalman filter. In the following example, we use the input-output data for the standard configuration of the test platform. The parameters of OKID algorithms are $p=200$ for OKID, $i=$ 100 for DP, DI, 2000 Markov parameters arranged in a 1000 by 1000 Hankel matrix for ERA and ERA-DC. Obviously, there is no significant difference for the singular values in the OKID/DI algorithm. In Figure 4, the singular values plots of OKID algorithms show that the first three-order vibration mode is the main component of system dynamics. In order to compare the performance of different OKID algorithms, the first six singular values are reserved to compute the system parameters. 


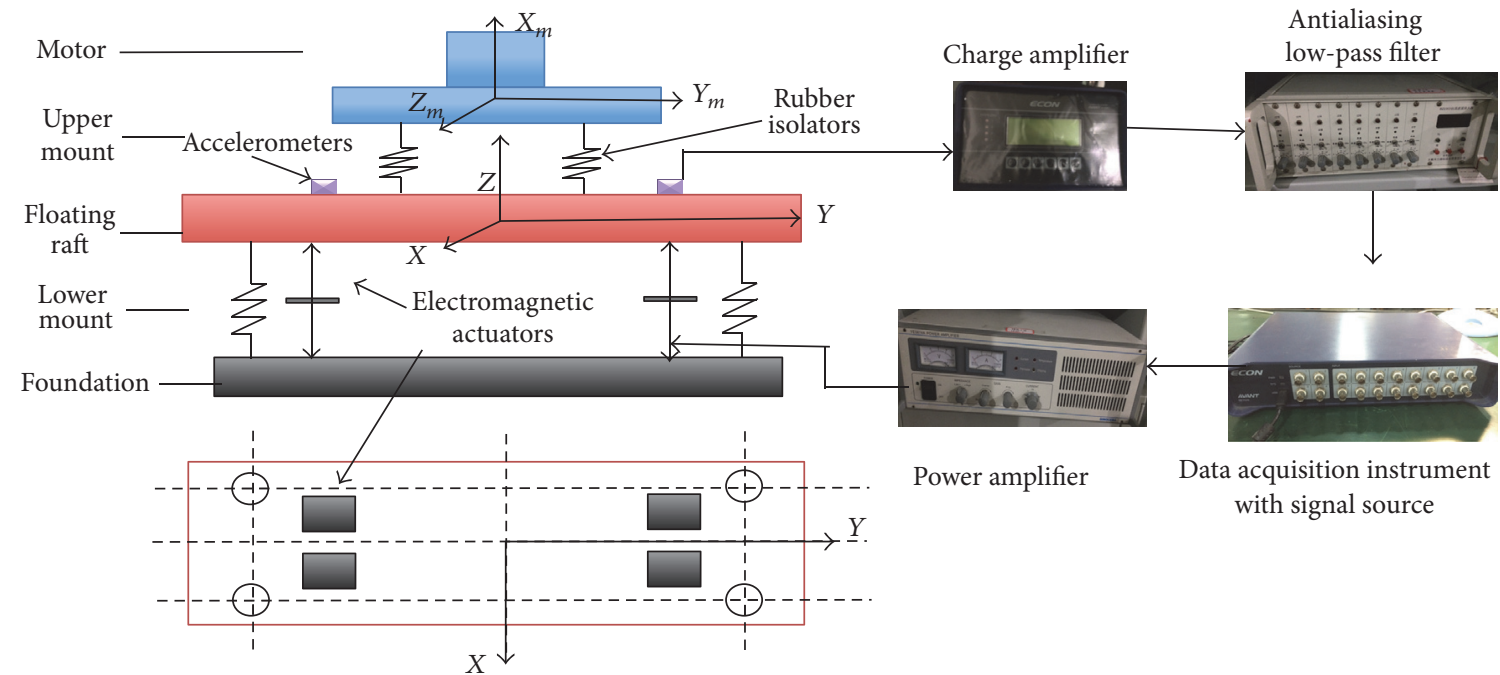

FIGURE 3: Structure diagram of floating raft experimental tests for input-output data.

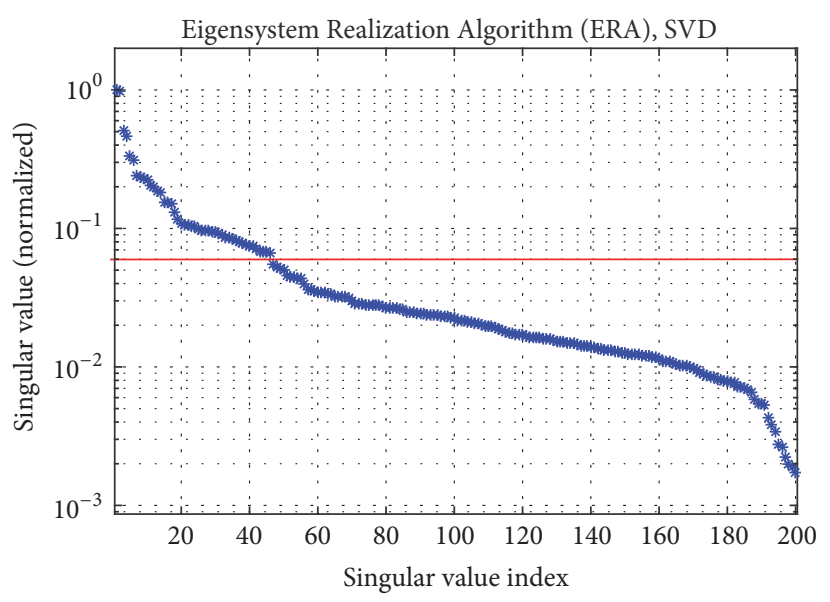

(a) OKID/ERA

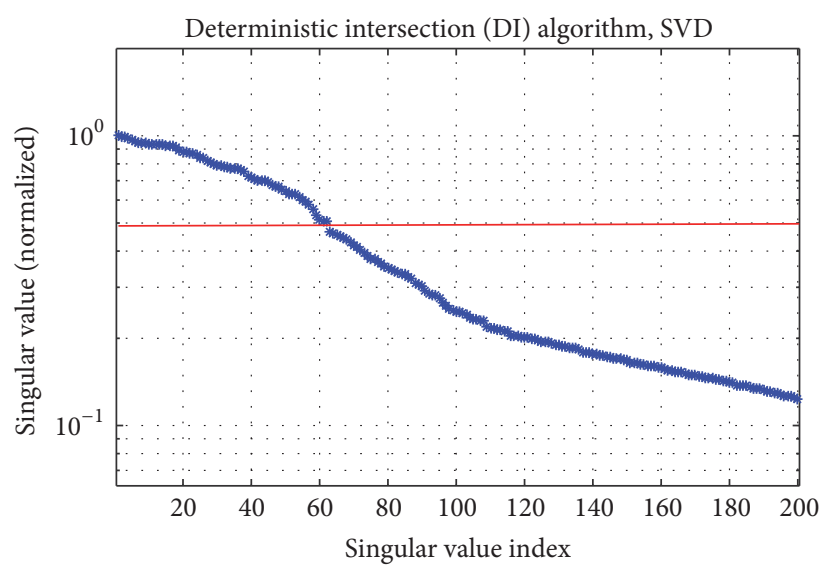

(c) $\mathrm{OKID} / \mathrm{DI}$

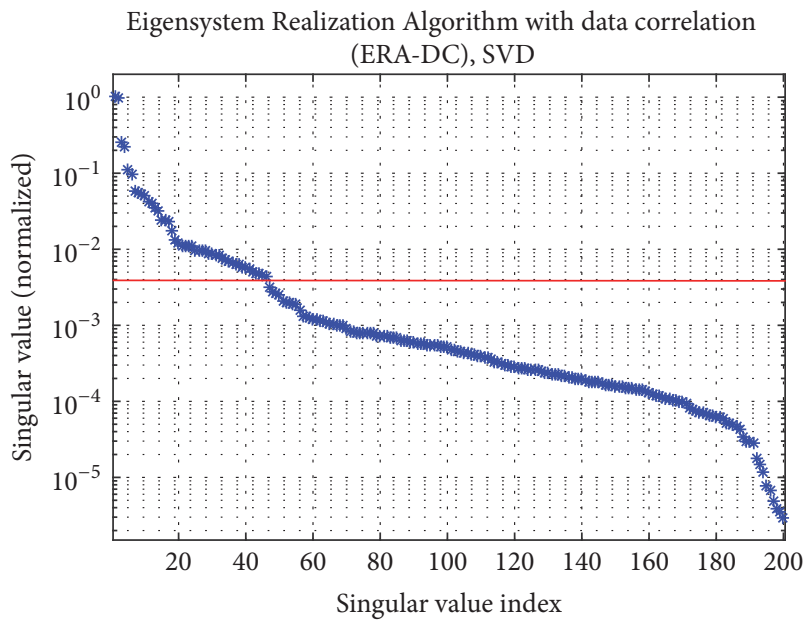

(b) OKID/ERA-DC

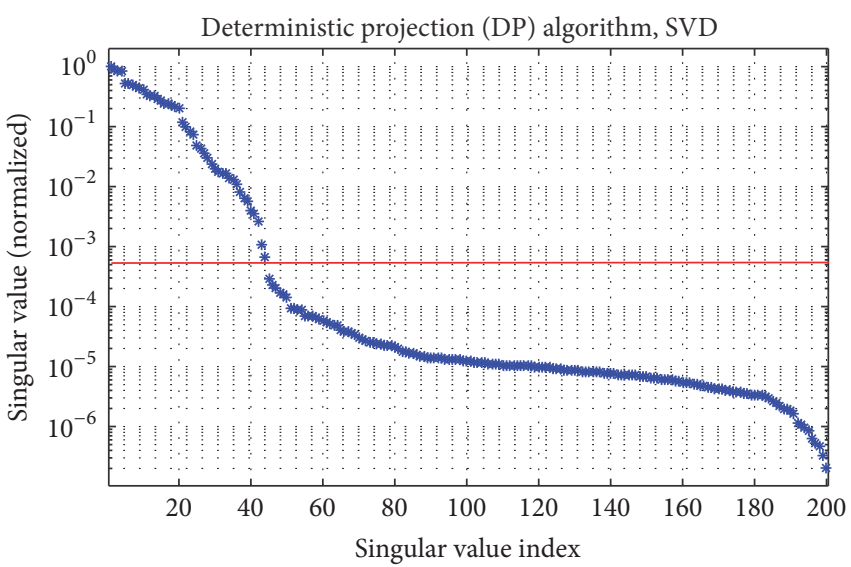

(d) $\mathrm{OKID} / \mathrm{DP}$

FIGURE 4: Singular values plots of the identification for floating raft platform (200 largest singular values). Algorithm parameters used in the identification: $p=200$ for OKID; $i=100$ for DP, DI; 2000 Markov parameters arranged in a 1000 by 1000 Hankel matrix for ERA and ERA-DC. 
TABLE 1: Identified natural frequencies $(\mathrm{Hz})$ and damping factors of the experimental structure choosing $n=6$ and corresponding output RMS error for both the identified system (S) and Kalman filter (K).

\begin{tabular}{lccccccrr}
\hline Method & $f_{1}$ & $\zeta_{1}$ & $f_{2}$ & $\zeta_{2}$ & $f_{3}$ & $\zeta_{3}$ & $\operatorname{err}_{\mathrm{RMS}}^{\mathrm{S}}$ & $\operatorname{err}_{\mathrm{RMS}}^{\mathrm{K}}$ \\
\hline Input-output data & 16.9 & 0.063 & 24.6 & 0.020 & 45.4 & 0.010 & \\
OKID/ERA & 17.0 & 0.045 & 24.8 & 0.017 & 45.6 & 0.008 & 0.0579 \\
OKID/ERA-DC & 17.0 & 0.045 & 24.8 & 0.017 & 45.6 & 0.008 & 0.0568 & 0.0452 \\
OKID/DI & 20.18 & 0.246 & 25.1 & 0.026 & 45.7 & 0.015 & 0.0826 & 0.0575 \\
OKID/DP & 17.3 & 0.065 & 25.0 & 0.019 & 45.5 & 0.007 & 0.0786 & 0.0453 \\
\hline
\end{tabular}

Retaining 6 largest singular values yields the modal parameters in Table 1 . In addition to OKID/DI, the other 3 methods give similar natural frequencies and damping factors, confirming the validity of the OKID approach. Table 1 also reports the root mean square (RMS) error between the actual output and the output predicted by identified system in (4a) and (4b), which is driven by the measured input. This corresponds to multistep-ahead prediction and the resulting output error is due not only to the identification error but also to the process and measurement noise affecting the data. In contrast, the RMS error with superscript $K$ refers to the error between the measured output and the output predicted by the identified Kalman filter in (19a) and (19b), which is driven by both the measured input and output. This corresponds to one-step-ahead prediction, since the past output values are taken into account in predicting the next output. Hence, the error of the Kalman filter is expected to be smaller. Indeed, the Kalman filter is the state-space model meant to minimize the LS error with respect to the measured output. The RMS error of output predicted by the identified Kalman filter over an independent set of data is then a good metric in this example to judge the performance of the different identification methods.

The identified model from OKID/DP algorithm is applied to get low order dynamic modeling of floating raft platform from input-output data. The order of the identified model can be obtained by the number of nonzero singular values for the deterministic system identification. However, for the stochastic identification, noise does not allow singular value decreases to zero. Therefore, the amplitude of singular values is sufficiently large relative to the others: namely, the number of large singular values is selected in the order of identified model. The plot of the singular values of $H_{0}$ in (16) is shown in Figure 4(d), a large gap between the zero and nonzero singular values characterizes the SVD plot of OKID/ERA, from which the system order was chosen to be 44 . The matrices $A \in \mathbb{R}^{44 \times 44}, B \in \mathbb{R}^{44 \times 2}, C \in \mathbb{R}^{2 \times 44}$, and $D \in \mathbb{R}^{2 \times 2}$ and observer gain $K \in \mathbb{R}^{44 \times 2}$ were then recovered by ERA.

Figure 5 depicts the predicted output in comparison with the real output and Figure 6 the estimated output in comparison with output data. The predicted output is the output restricted from identified model only, whereas the estimated output is the output reconstructed from identified observer. There are visible differences in the predicted and estimated outputs. Comparison of observer output with measured response shows extremely good agreement, indicating that the observer is correcting for the system uncertainties including nonlinearities.

Table 2 presents the pulse response weighting indices of identified space-state model. The first row is simply an integer denoting the stat element in a certain coordinate representation. This number runs from 1 to the order of unreduced system which is 44 . The second row contains the weighting indices that measure the relative contribution of every element to the overall dynamics. The weighting values are not arranged in any particular order, but they tend to drop off as a function of state element. The largest 20 order state elements suggest that there are a few dominant modes followed by a large number of less significant modes where the cut-off is not quite obvious. The matrices of full model $A \in \mathbb{R}^{44 \times 44}, B \in \mathbb{R}^{44 \times 2}, C \in \mathbb{R}^{2 \times 44}$, and $D \in \mathbb{R}^{2 \times 2}$ and observer gain $K \in \mathbb{R}^{44 \times 2}$ were reduced to reduced-order model $A r \in \mathbb{R}^{20 \times 20}, B r \in \mathbb{R}^{20 \times 2}, C r \in \mathbb{R}^{2 \times 20}$, and $D r \in \mathbb{R}^{20 \times 2}$ and observer gain $K r \in \mathbb{R}^{20 \times 2}$.

Recall that, for a linear system, the response to any general input is simply a convolution sum of that input with the system unit pulse response. Hence, one way to quantify the accuracy of the reduced-order model is by comparing its unit pulse response with that of the full model. This pulse response error comes out to be $1.2736 \%$. It also asked us if we would like to see comparison between the frequency response functions of the reduced model and those of the full model. Since this is a two-input two-output system, there will be 4 sets of frequency response function (magnitude and phase), one for each input-output pair. Figure 7 shows the comparison of frequency response function for the floating raft system between full model identified via OKID and reduced model. The two models based on input-output data provide very similar FRF; close agreement of FRF between full model and reduced model confirms the validity of the design approach.

Besides checking the frequency response functions, we can also validate the reduced-order model by comparing the pulse response of the reduced model with that of the original model. Figure 8 reports the comparison of the pulse responses for the floating raft system between full model identified via OKID and reduced model. These figures show that reduced model results agree well with the full model. Note that the pulse responses of the reduced-order model and the full order model practically overlap, which little information is lost by reducing the model order by this method. Of course, the 20th model size to retain is based on trade-off analysis between the model accuracy and the computational cost incurred during 

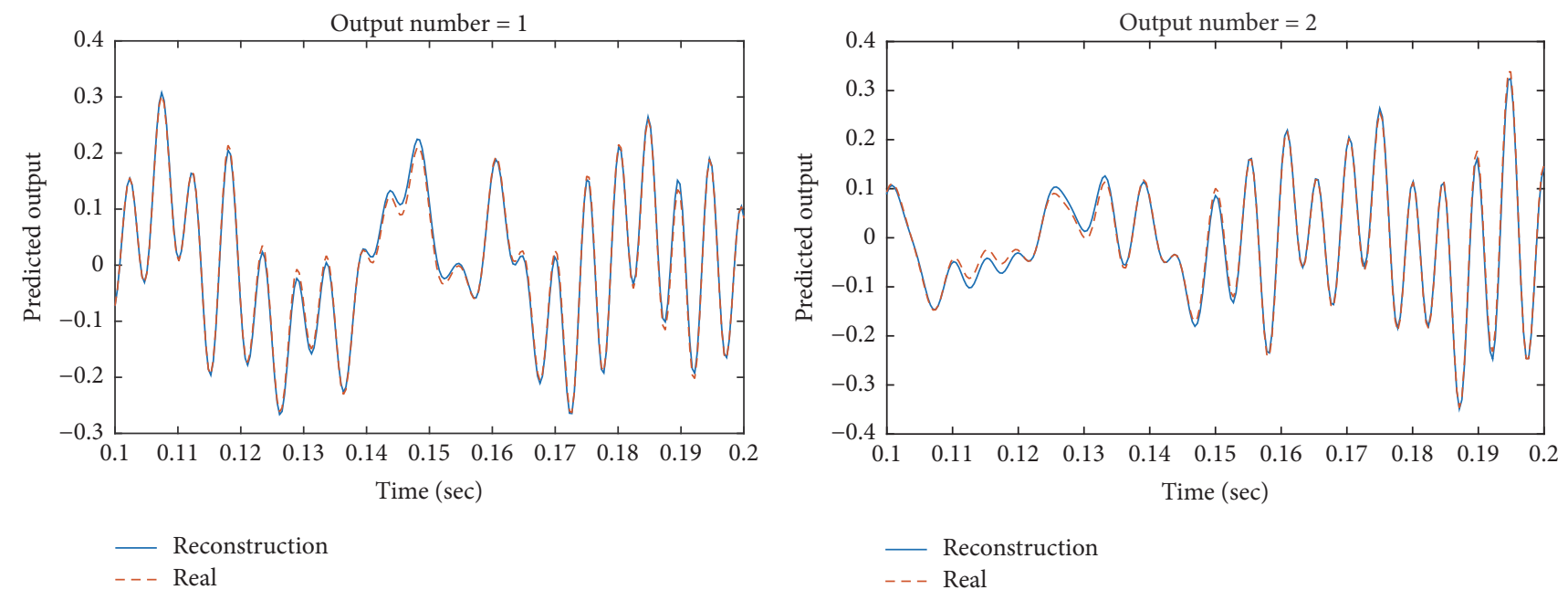

FIGURE 5: Comparison of real output with predicted output.
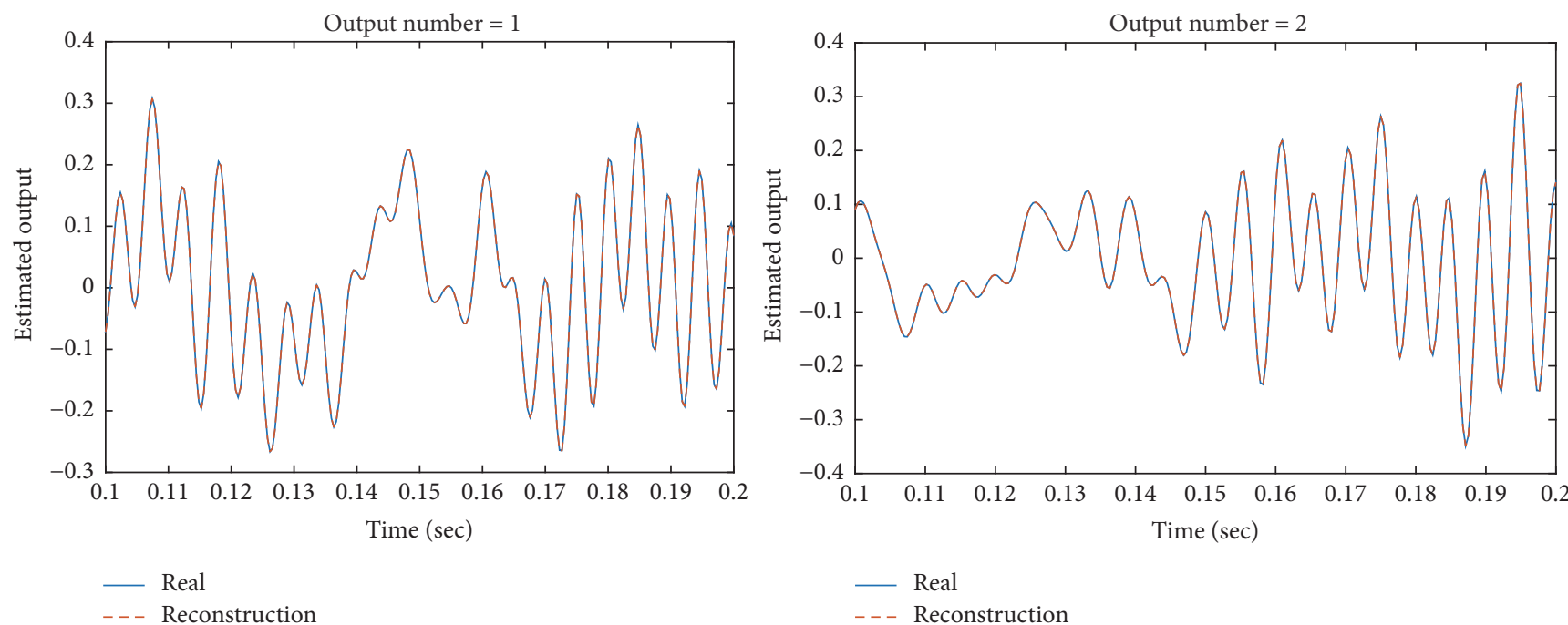

FIgURE 6: Comparison of real output with estimated output.

the control implementation process. Both FRF and pulse response results confirm the validity and the benefits of the approach.

\section{Controller}

4.1. $H_{\infty}$ Control Algorithm. The following simulation and control experiment aim to illustrate the correctness and effectiveness of the space-state model from OKID approach proposed in this paper. Floating raft systems are generally subject to multiple frequency varying vibrations.

$H_{\infty}$ control algorithm is one of feedback control algorithms, where no reference signal for control operation can effectively suppress multifrequency varying vibration; the block diagram of feedback control is shown in Figure 9. The error signal $e$ is produced by the superposition of the disturbance signal $d$ and the desired output signal $y$. Then $e$ is measured by the sensor and transmitted to the controller $K(s)$.
According to the control law, the control signal $u$ is calculated by the controller, then it goes through the control channel $G(s)$ to generate the desired output signal $y$. Essentially, the disturbance signal $d$ is canceled around the superposition point by generating and combining the desired output signal $y$ canceling noise the frequency varying vibration.

Sensitivity function $S$ is an important indicator of the sensitivity, which reflects the performance of the closed-loop control system and the relative stability of the system. In the block diagram, the transfer function of the disturbance $d$ to the output $y$ is defined as $S$. The magnitude of the sensitivity function $S$ represents the ability to suppress disturbance. The expression of $S$ is as follows:

$$
S(s)=\frac{1}{1+K(s) G(s)} .
$$

In the Nyquist diagram, the sensitivity of the system $S(j w)$ is analyzed based on the value of $K(j w) G(j w) . \rho$ is 
TABle 2: Pulse response weighting indices.

\begin{tabular}{|c|c|}
\hline Number & Relative value \\
\hline 1 & $1.28 e-01$ \\
\hline 2 & $1.29 e-01$ \\
\hline 3 & $9.87 e-02$ \\
\hline 4 & $1.52 e-02$ \\
\hline 5 & $9.85 e-02$ \\
\hline 6 & $4.61 e-02$ \\
\hline 7 & $1.20 e-02$ \\
\hline 8 & $3.21 e-02$ \\
\hline 9 & $5.62 e-02$ \\
\hline 10 & $2.64 e-02$ \\
\hline 11 & $7.59 e-03$ \\
\hline 12 & $9.45 e-04$ \\
\hline 13 & $2.43 e-03$ \\
\hline 14 & $2.39 e-03$ \\
\hline 15 & $4.64 e-03$ \\
\hline 16 & $5.59 e-03$ \\
\hline 17 & $6.36 e-03$ \\
\hline 18 & $1.17 e-03$ \\
\hline 19 & $2.23 e-04$ \\
\hline 20 & $7.31 e-04$ \\
\hline 21 & $3.23 e-03$ \\
\hline 22 & $7.98 e-04$ \\
\hline 23 & $1.57 e-04$ \\
\hline 24 & $1.73 e-04$ \\
\hline 25 & $1.05 e-04$ \\
\hline 26 & $9.96 e-04$ \\
\hline 27 & $5.39 e-04$ \\
\hline 28 & $9.22 e-04$ \\
\hline 29 & $3.67 e-04$ \\
\hline 30 & $4.60 e-05$ \\
\hline 31 & $3.60 e-05$ \\
\hline 32 & $1.24 e-04$ \\
\hline 33 & $7.13 e-06$ \\
\hline 34 & $4.25 e-06$ \\
\hline 35 & $2.66 e-07$ \\
\hline 36 & $1.03 e-05$ \\
\hline 37 & $1.69 e-07$ \\
\hline 38 & $6.26 e-07$ \\
\hline 39 & $6.59 e-08$ \\
\hline 40 & $5.13 e-08$ \\
\hline 41 & $5.13 e-08$ \\
\hline 42 & $7.69 e-07$ \\
\hline 43 & $1.71 e-08$ \\
\hline 44 & $1.97 e-08$ \\
\hline
\end{tabular}

the shortest distance from $K(j w) G(j w)$ to point $(-1,0)$; the expression of $\rho$ is as follows:

$$
\rho=\min |1+K(j w) G(j w)| .
$$

The distance $\rho$ is related to the maximum amplitude of the sensitivity function $M_{s}$,

$$
M_{s}=\max |S(j w)|=\frac{1}{\rho},
$$

where $M_{s}$ is the maximum amplitude of the sensitivity function. Therefore, lower magnitude of $M_{s}$ is designed to get improved ability to suppress disturbance in the design process of $H_{\infty}$ controller.

The difference between the identified model and the actual controlled object must be taken into consideration when designing the control system, so that the designed controller based on an error model can still ensure the actual closed-loop system's stability and achieve the desired designing goals. The difference between the identified model and the controlled object is called model uncertainty, and the common description methods for model uncertainty are addition uncertainty description and multiplication uncertainty description, respectively, as shown in

$$
\begin{aligned}
& \widetilde{G}(s)-G(s)=\Delta(s) * W_{2}(s), \\
& \frac{\widetilde{G}(s)-G(s)}{G(S)}=\Delta(s) * W_{3}(s),
\end{aligned}
$$

where $\widetilde{G}(s)$ is the actual controlled object, $G(s)$ is the nominal model, $\Delta(s)$ is the uncertainty after normalized, $W_{2}(s)$ is additive uncertainty of model, and $W_{3}(s)$ is multiplicative uncertainty of model.

The robust stability requirement and the performance requirement of the closed-loop control system should be considered for designing $H_{\infty}$ controller. The mixed sensitivity problem is a typical designing problem for $H_{\infty}$ controller, which can transform the requirements into the optimization problem of $H_{\infty}$ norm [26]. A typical block diagram of the mixed sensitivity problem is shown in Figure 10, where $G(s)$ is the nominal model, $K(s)$ is the controller to be designed, $W_{1}$, $W_{2}$, and $W_{3}$ are three different weighting functions, $w$ is the input signal, $z_{1}, z_{2}$, and $z_{3}$ are the weighted outputs, $y$ is the error signal measured by sensor, and $u$ is the control output signal of the controller. The area surrounded by the dashed box is augmented controlled object, including the controlled object $G(s)$ and three weighting functions.

The mixed sensitivity problem can be solved by selecting three appropriate weighting functions in the design of the controller $K(s)$ to meet the system's performance and stability requirements: namely, the controller $K(s)$ enables the establishment of formula (29); the details of the mixed sensitivity problem can be found in [28]

$$
\left\|\begin{array}{l}
W_{1} S \\
W_{2} R \\
W_{3} T
\end{array}\right\|=\left\|\begin{array}{c}
\frac{W_{1}}{1+G K} \\
\frac{W_{2} K}{1+G K} \\
\frac{W_{3} G K}{1+G K}
\end{array}\right\| \leq 1,
$$

where $S$ is the sensitivity function, $R$ is the controller sensitivity function, and $T$ is the complementary sensitivity function. 

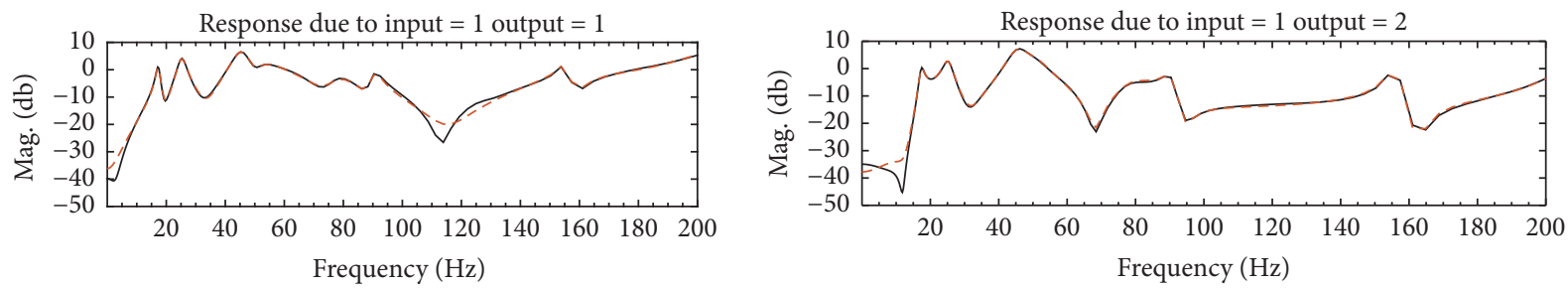

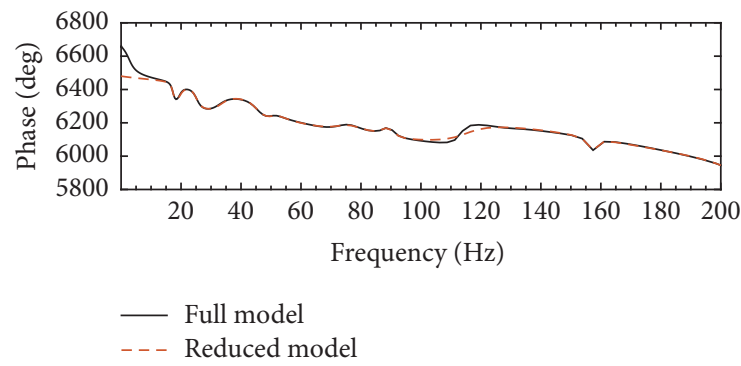

(a) FRF of 1st input-1st output
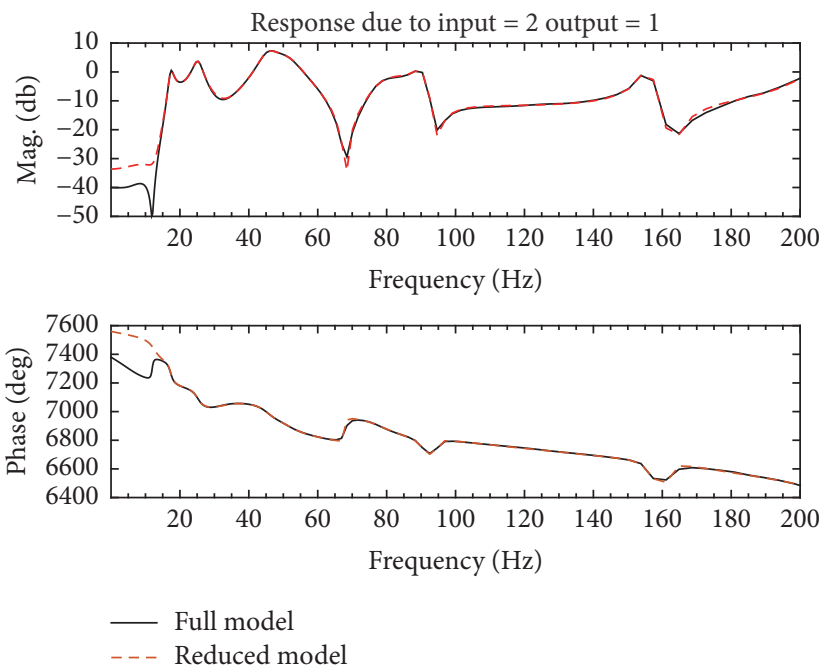

(c) FRF of 2nd input-1st output

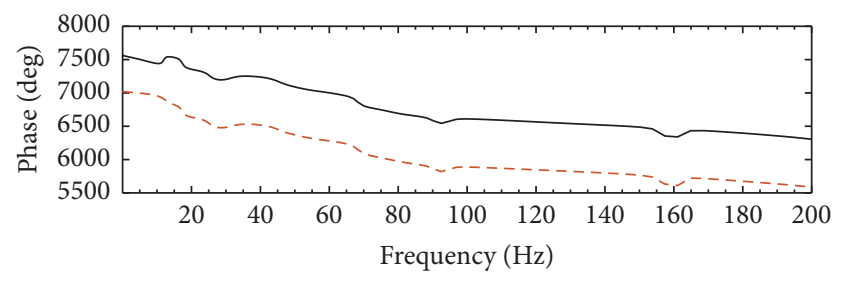

- Full model

- - - Reduced model

(b) FRF of 1st input-2nd output
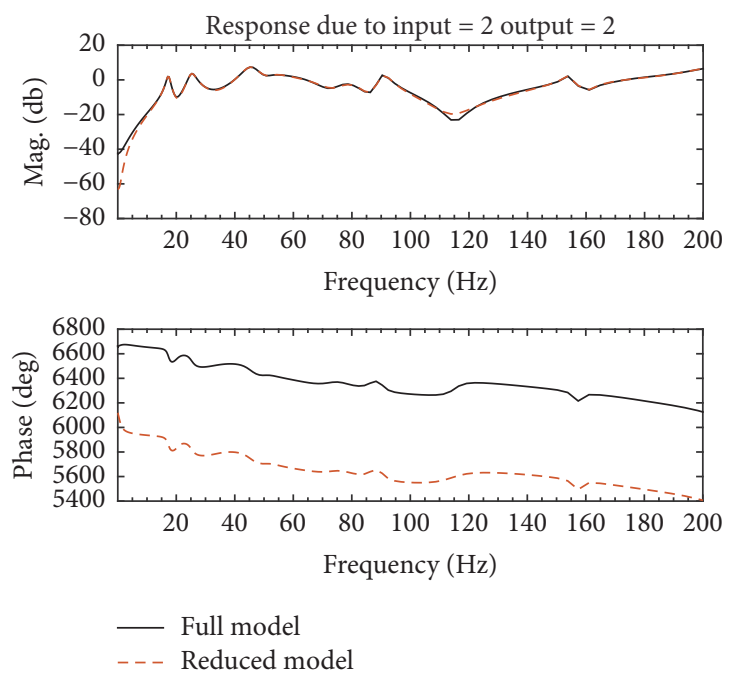

(d) FRF of 2nd input-2nd output

FIGURE 7: Comparison of the FRF (frequency response function) for the floating raft system between full model identified via OKID and reduced model.

And $\left\|W_{1} S\right\| \leq 1$ represents the performance requirements; $\left\|W_{2} R\right\| \leq 1$ and $\left\|W_{3} T\right\| \leq 1$ both represent the robust stability requirements.

The solution of mixed sensitivity problem needs to be converted into a standard $H_{\infty}$ control problem. The standard $H_{\infty}$ control problem can be solved by designing the controller $K(s)$ according to the given augment matrix $P(s)$ to meet the performance requirements of the system and the stability of the closed-loop control system. The block diagram of the standard $H_{\infty}$ controller is shown in Figure 11

$$
\begin{aligned}
\dot{x} & =A x+\left[\begin{array}{ll}
B_{1} & B_{2}
\end{array}\right]\left[\begin{array}{l}
w \\
u
\end{array}\right], \\
{\left[\begin{array}{l}
z \\
y
\end{array}\right] } & =\left[\begin{array}{l}
C_{1} \\
C_{2}
\end{array}\right] x+\left[\begin{array}{ll}
D_{11} & D_{12} \\
D_{21} & D_{22}
\end{array}\right]\left[\begin{array}{l}
w \\
u
\end{array}\right], \\
\left\|T_{z w}\right\| & \leq \gamma,
\end{aligned}
$$

where $T_{z w}$ is the closed-loop transfer function of $w$ to $z$. In the solution process of controller $K(s)$, the value of $\gamma$ should be approximately equal to 1 .

The solutions of the standard $H_{\infty}$ controller can be summarized as Riccati method, maximum principle method, differential game method, and so forth. One of the most successful solutions for Riccati method relies on two Riccati equations to efficiently compute one solution of standard $H_{\infty}$ controller. Providing a comprehensive theoretical deduction is beyond the scope of the present paper; Karimi and Emedi have offered a classic Riccati method to solve the standard $H_{\infty}$ control problem [29]. In the paper, the standard $H_{\infty}$ controller is solved by Riccati method by robust control toolbox in the MATLAB software.

4.2. $H_{\infty}$ Controller. The solution of mixed sensitivity problem requires prior to select the three appropriate weighting functions $W_{1}, W_{2}$, and $W_{3}$, since the quality of these three 


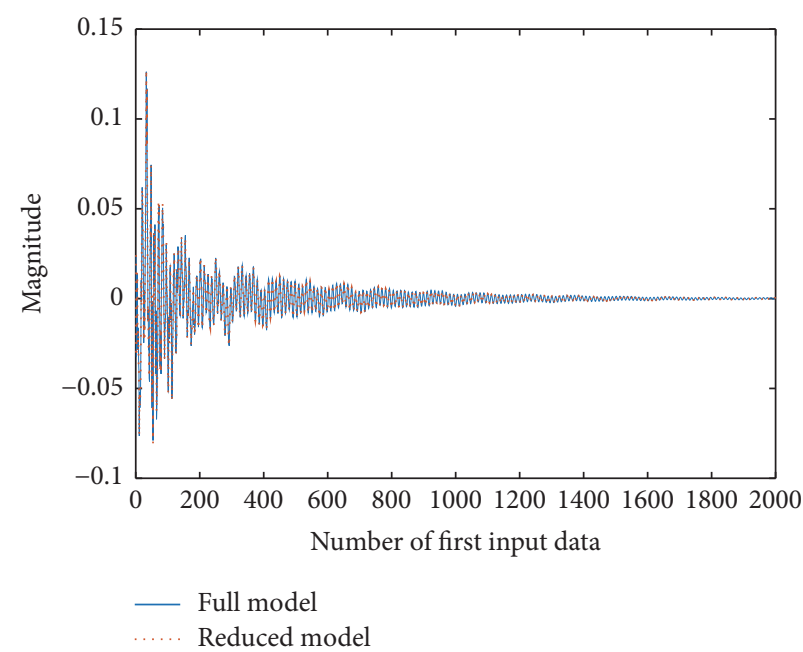

(a) Pulse response of 1st input-1st output

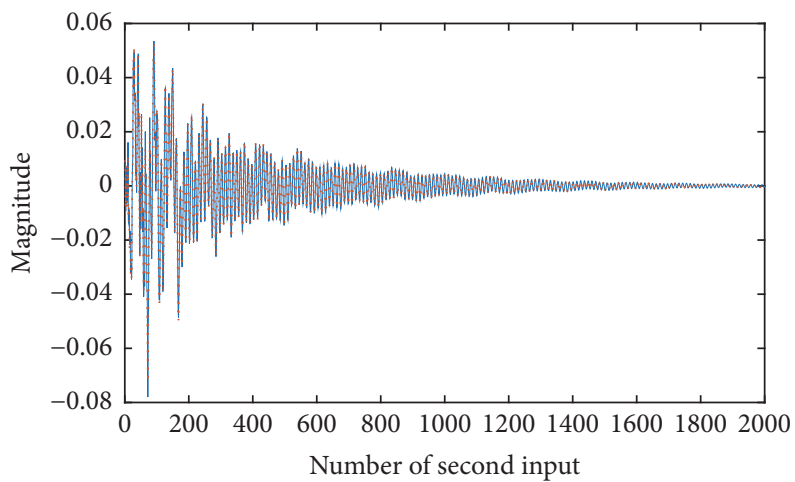

Full model

Reduced model

(c) Pulse response of 2nd input-1st output

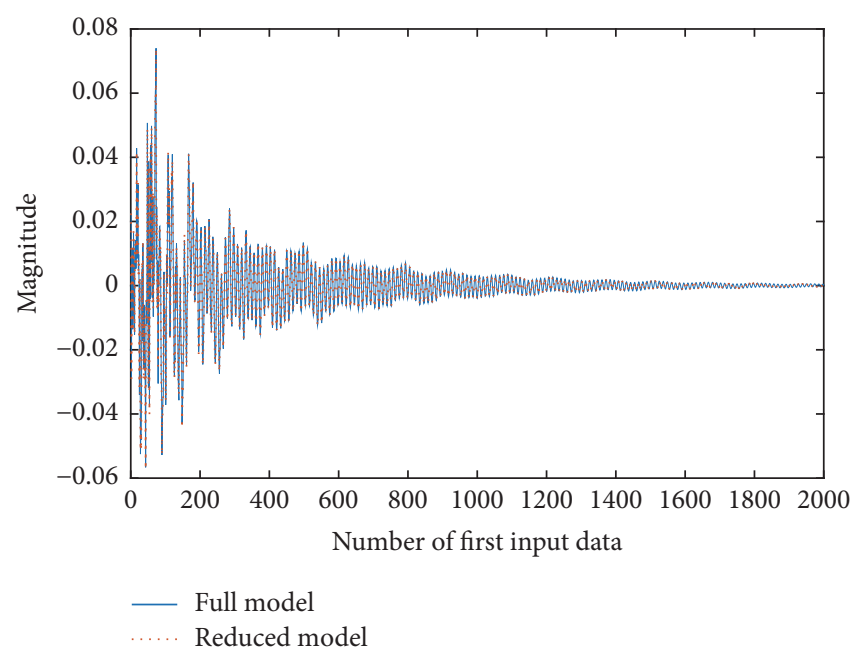

(b) Pulse response of 1st input-2nd output

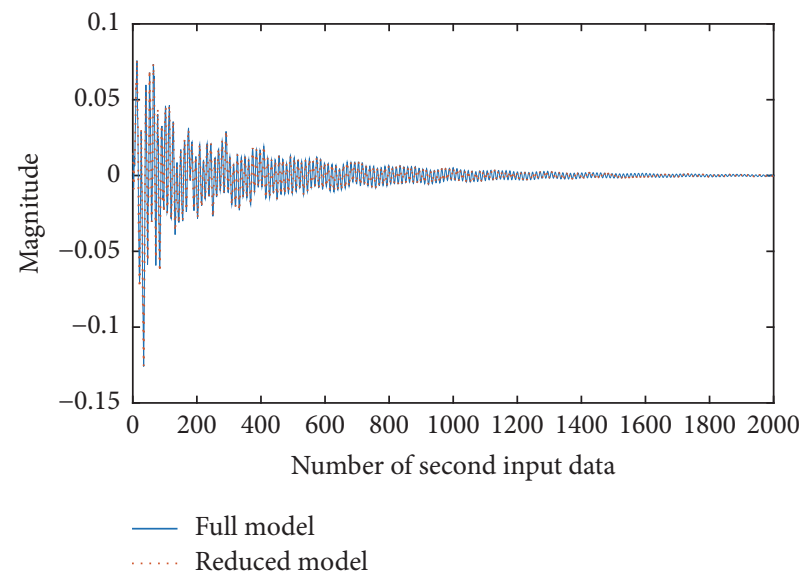

(d) Pulse response of 2nd input-2nd output

FIGURE 8: Comparison of the pulse responses for the floating raft system between full model identified via OKID and reduced model.

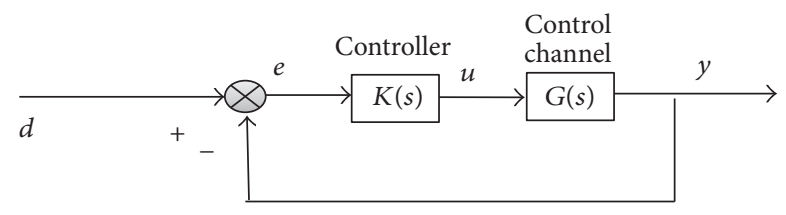

FIGURE 9: Block diagram of feedback control.

weighting functions directly affects the designed controller whether to meet the designing requirements. The weighting functions $W_{2}$ and $W_{3}$ are selected first and then the weighting function $W_{1}$. The weighting functions $W_{2}$ and $W_{3}$ are related to model uncertainties, including the absolute error and the relative error between the model response and the experimental frequency response, where $W_{2}$ is an index of describing the model uncertainty characteristics in the low and middle frequency ranges and $W_{3}$ is in the high frequency ranges. In this paper, the control object is a dual-frequency varying signal in the low and middle frequency ranges, and the identified space-state model and experimental frequency responses are limited from 0 to $200 \mathrm{~Hz}$, and the absolute

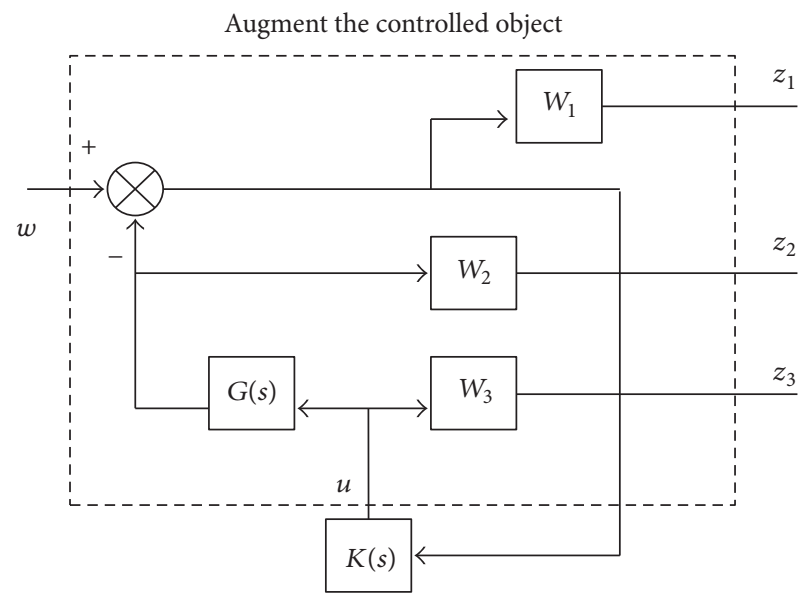

FIGURE 10: Block diagram of the mixed sensitivity problem.

errors are all less than 0.2 . In order to obtain a smaller order and higher robustness controller, the optional weighting function $W_{2}$ is assigned to $0.5 ; W_{3}$ is 0 . 


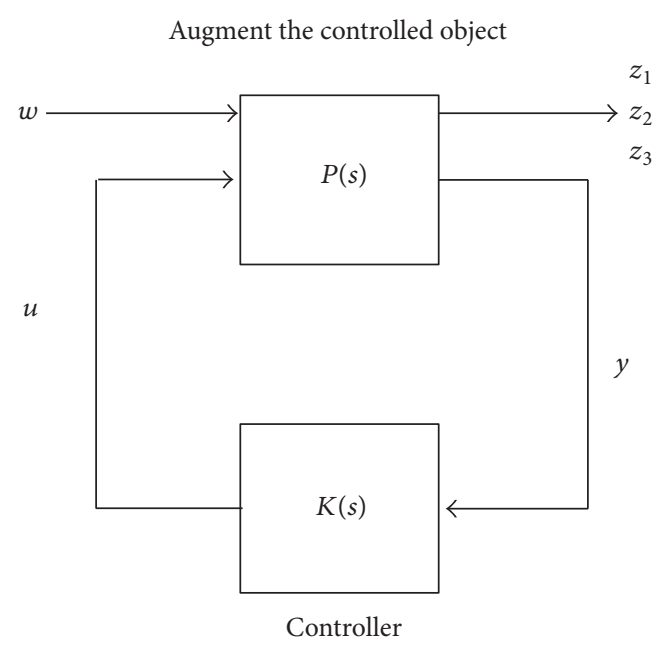

FIGURE 11: Block diagram of the standard $H_{\infty}$ controller.

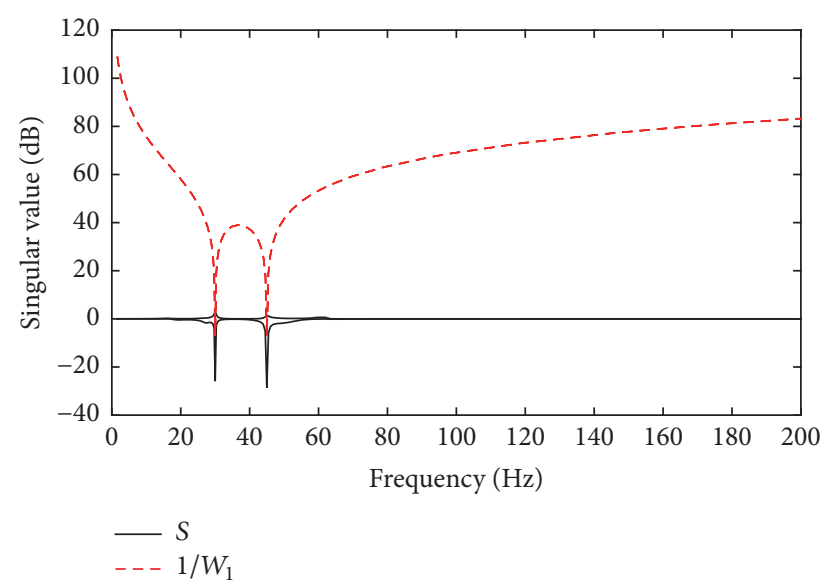

FIGURE 12: Shaping design result for dual-dominant frequency varying disturbance.

The weighting function $W_{1}$ should be selected according to the frequency characteristics of external interference. The design purpose of $H_{\infty}$ controller is to suppress the frequency varying frequency vibration, whose dominant frequency ranges from $1 \mathrm{~Hz}$. Therefore, the amplitude of the performance-related sensitivity function $S$ in dominant frequency must be less than 1 . The system performance requirement can be described by

$$
\left\|W_{1} S\right\| \leq 1 \Longrightarrow|S(j w)| \leq\left|W_{1}^{-1}(j w)\right| .
$$

Equation (31) shows frequency characteristic of the sensitivity function $S$, where $|S(j w)|$ must lie below $\left|W_{1}^{-1}(j w)\right|$ and the amplitude of $\left|W_{1}(j w)\right|$ at dominant frequency must be larger than 1. Both $|S(j w)|$ and $\left|W_{1}(j w)\right|$ curves produce a depression at the dominant frequency, where the feedback control system can highly suppress the disturbance signal at the dominant frequency.

In the paper, the dual-dominant frequency disturbances are the objects of the controller design; thus, $W_{1}$ should be selected as bimodal shape

$$
\begin{aligned}
& W_{1}=k s^{2} \frac{1}{\left(s^{2}+2 w_{n 1} \xi_{1} s+w_{n 1}^{2}\right)\left(s^{2}+2 w_{n 2} \xi_{2} s+w_{n 2}^{2}\right)}, \\
& w_{d 1}=w_{n 1} \sqrt{1-\xi_{1}^{2}}, \\
& w_{d 2}=w_{n 2} \sqrt{1-\xi_{2}^{2}},
\end{aligned}
$$

where $w_{d 1}$ and $w_{d 2}$ are the dominant frequency to be suppressed and $\xi_{1}, \xi_{2}$, and $k$ are adjustment parameters.

The dominant frequencies of excitation source to be suppressed are, respectively, $30 \mathrm{~Hz}$ and $45 \mathrm{~Hz}$, where the variation range of frequency is no more than $1 \mathrm{~Hz}$. The weighting function $W_{1}$ is executed with adjusting the parameters $\xi_{1}=$ $0.0005, \xi_{2}=0.0005$, and $k=80$ according to (32). In order to get $H_{\infty}$ controller for 2 inputs/ 2 outputs dual-dominant frequency varying vibrations, the reduced state-space model is applied to the robust control toolbox. Then, the function "hinfsyn" in the MATLAB software is used to solve $H_{\infty}$ controller, which has the solutions and ensures that the value of $\gamma$ should be approximately equal to $1, \gamma=1.0371$. The results from numerical algorithm for the weighting function are eventually identified as

$$
\begin{aligned}
& W_{2}=\left[\begin{array}{cc}
0.5 & 0 \\
0 & 0.5
\end{array}\right], \\
& W_{3}=0 .
\end{aligned}
$$

The shaping design diagram for dual-dominant frequency varying vibration is shown in Figure 12, which reports that two singular value curves of sensitive function
$S$ are below the weighting function curves $W_{1}^{-1}$. Meanwhile, both curves have depressions at and near the $30 \mathrm{~Hz}$ and $45 \mathrm{~Hz}$, which indicate that the closed-loop control system can 


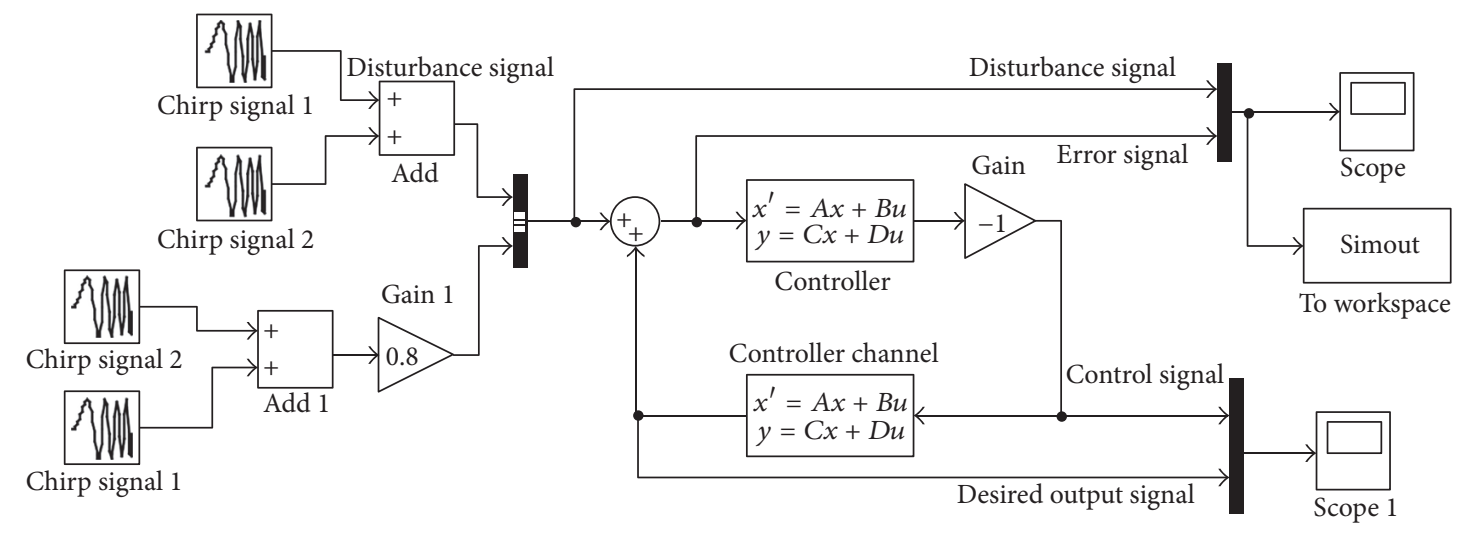

FIGURE 13: The simulation block diagram of $H_{\infty}$ control system for MIMO dual-dominant frequency varying disturbance in the Simulink software.

effectively suppress dual-dominant frequency varying vibration.

4.3. Simulation. Fuzzy control algorithm has the ability to handle numeric and linguistic knowledge simultaneously, and it is not sensitive to the variation of process parameters. A fuzzy controller based on minimization of force transmissibility is proposed to reduce the vibration of floating raft system. The results show that the proposed fuzzy controller has much better performance in vibration isolation than PID controller and passive system [35]. In this paper, in order to verify the validity of the proposed improvements, mathematical models of the system are identified by OKID from input-output measured data of floating raft system. Then, $H_{\infty}$ control algorithm and fuzzy control algorithm are applied independently to the active vibration isolation platform under the same conditions of excitation. Finally, we compare the performance of $H_{\infty}$ control algorithm and the performance of the fuzzy control algorithm with the same model obtained by OKID.

The following simulations in Simulink software aim to illustrate the correctness and effective of $H_{\infty}$ controller for dual-dominant frequency varying vibration proposed in this paper. Figure 13 reports simulation of designed $H_{\infty}$ controller for the MIMO system. The disturbance signal consists of two sweep signals, where dominant frequencies are $30 \mathrm{~Hz}$ and $45 \mathrm{~Hz}$, respectively, with each varying range equal to $\pm 1 \mathrm{~Hz}$ and RMS (root mean square) $1 \mathrm{~V}$. The other disturbance signal has the same frequency characteristics and RMS $0.8 \mathrm{~V}$. The reduced state-space model identified from OKID and $H_{\infty}$ controller are executed into the simulation block diagram. The main design parameters of $H_{\infty}$ algorithm in the Simulink are as follows: ode8 (Dormand-Prince) is selected as fixedstep solver and fixed-step size is 0.0001 ; the simulation time is $0-5 \mathrm{~s}$.

Figure 14 shows the simulation block diagram in action before and after turning on $H_{\infty}$ control algorithm and fuzzy control algorithm. In the time domain diagram, compared with the passive signal, both the magnitude of $1 \#$ error signal and 2\# error signal gradually decreases from $2 \mathrm{~m} / \mathrm{s}^{2}$ to nearly $0.5 \mathrm{~m} / \mathrm{s}^{2}$ and becomes stable after $2 \mathrm{~s}$ when fuzzy controller is turned on. Correspondingly, $H_{\infty}$ controller has the same control process with fuzzy control. Further, $H_{\infty}$ controller has a better performance in the convergence speed and vibration reduction than fuzzy control for the dualdominant frequency varying disturbance. More specifically, in the frequency domain diagram, the amplitude of $1 \#$ error signal for $H_{\infty}$ controller and fuzzy control at $29.98 \mathrm{~Hz}$ decreases, respectively, from $0.8587 \mathrm{~m} / \mathrm{s}^{2}$ to $0.02923 \mathrm{~m} / \mathrm{s}^{2}$ and $0.4539 \mathrm{~m} / \mathrm{s}^{2}$, which have, respectively, the improvement up to $29.3 \mathrm{~dB}$ and $5.5 \mathrm{~dB}$ compared to the passive system. For the main component of the $1 \#$ error signal is at $45.01 \mathrm{~Hz}$, $H_{\infty}$ controller and fuzzy control have an improvement up to $25 \mathrm{~dB}$ and $13.9 \mathrm{~dB}$ when compared to the passive system. The simulation results show that $H_{\infty}$ control algorithm dramatically improves over the performance of the fuzzy control algorithm and passive system.

4.4. Experiment. Theoretical analysis and computer simulation are important but not sufficient for investigating the usefulness of new results, as practical factors such as measurement noise and actuators saturation are neglected in the theoretical and simulation analysis. The ultimate justification for the value and applicability of the proposed controller lies in the actual hardware implementation. To examine the performance of the proposed control solution, experimental tests are presented in this section.

Figure 15 presents structure diagram of floating raft experimental tests platform. Active-passive vibration isolation floating raft platform consists of the following components: (1) motors (vibration source), (2) upper mount system including 16 rubber isolators and upper base, (3) floating raft, (4) lower mount system including 4 rubber isolators and four electromagnetic actuators, and (5) foundation and $\mathrm{T}$ slot rigid base. Four active electromagnetic actuators and four rubber isolators are installed under the raft frame. The entire system rests on T-slot rigid foundation to minimize extraneous source of unmeasured excitation from being introduced through the base of the system. Four accelerometers attached at the installation point of electromagnetic actuators on the top of floating raft to measure acceleration signals. The floating raft has three dofs: one translational motion in the vertical direction $(Z)$ and two rotational motions, roll $\left(\Theta_{x}\right)$ and pitch $\left(\Theta_{y}\right)$. The translational motion in the vertical 

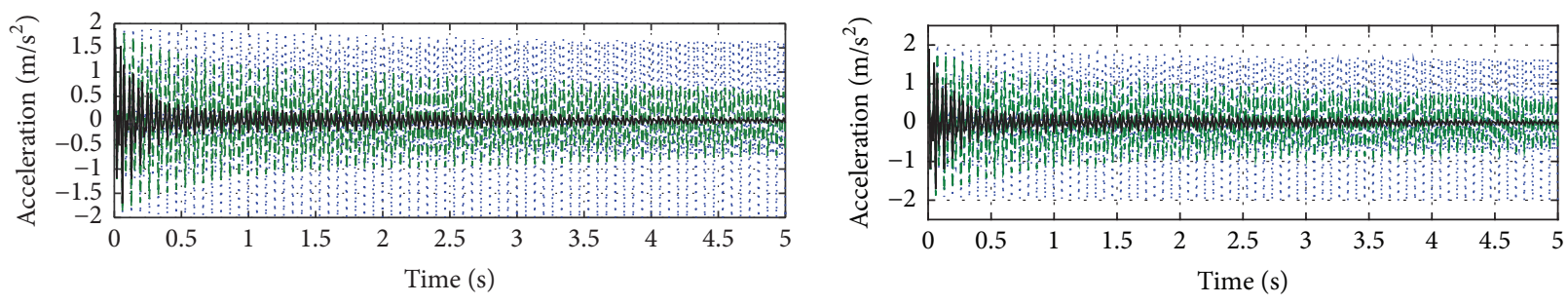

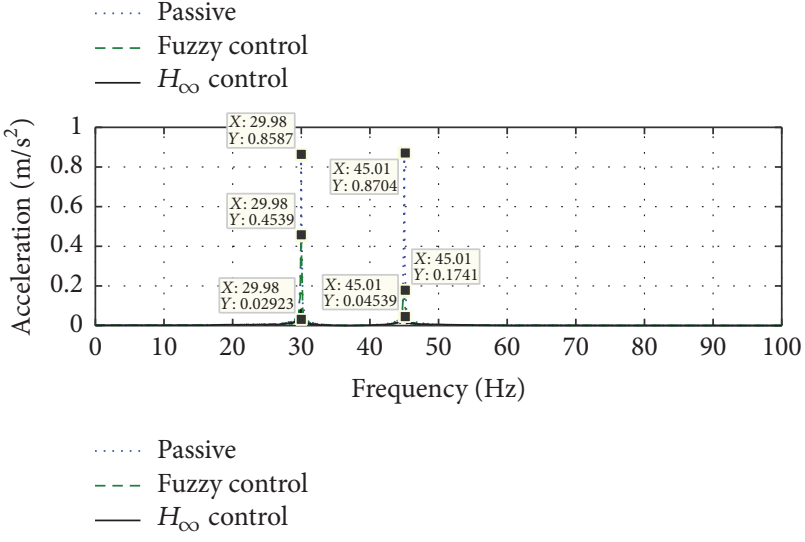

(a) 1\# error signal

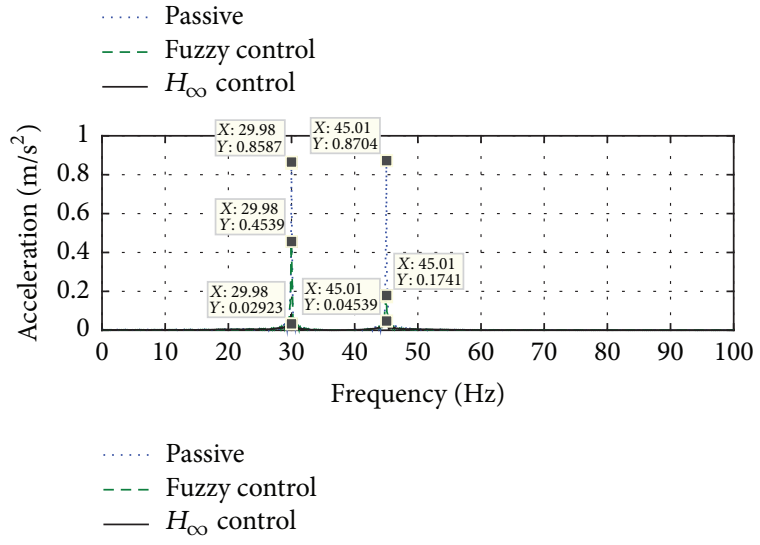

(b) 2\# error signal

FIGURE 14: The time-frequency simulation comparison charts of error signals between passive, fuzzy control, and $H_{\infty}$ control.

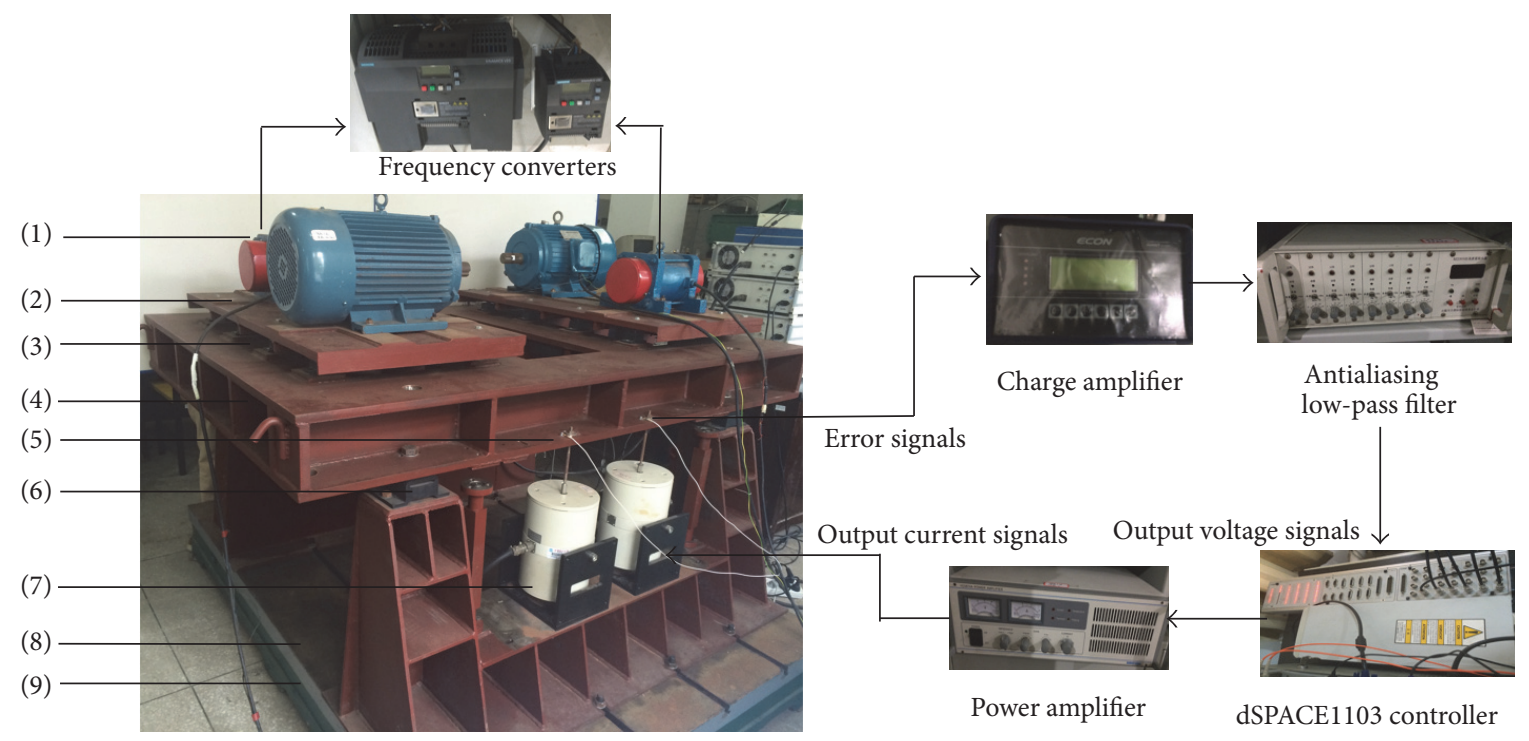
(1) Motors
(6) Lower rubbers
(2) Upper base
(7) Electromagnetic actuators
(3) Upper rubbers
(8) Foundation
(4) Floating raft
(9) T-slot rigid base
(5) Accelerometers

FIGURE 15: The structure diagram of $H_{\infty}$ control for the floating raft system. 


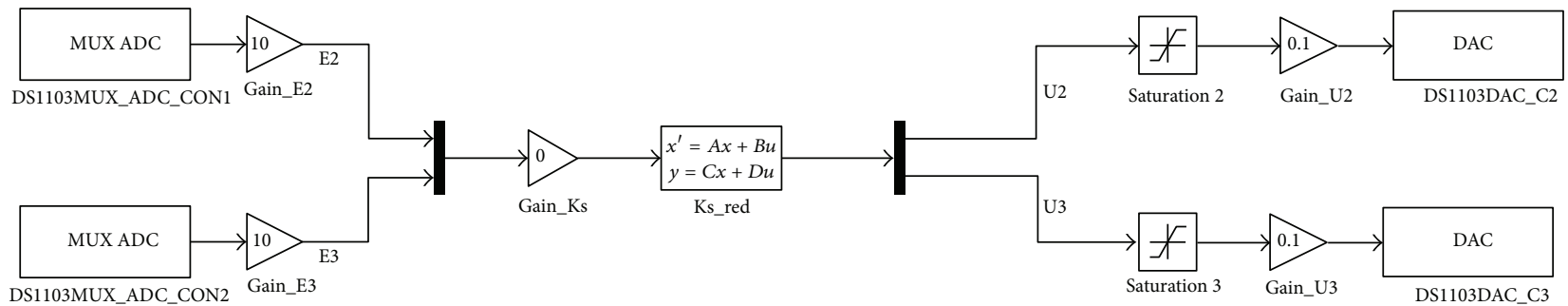

FIGURE 16: The picture of $H_{\mathrm{\infty}}$ control algorithm block diagram for the dSPACE1103 controller.
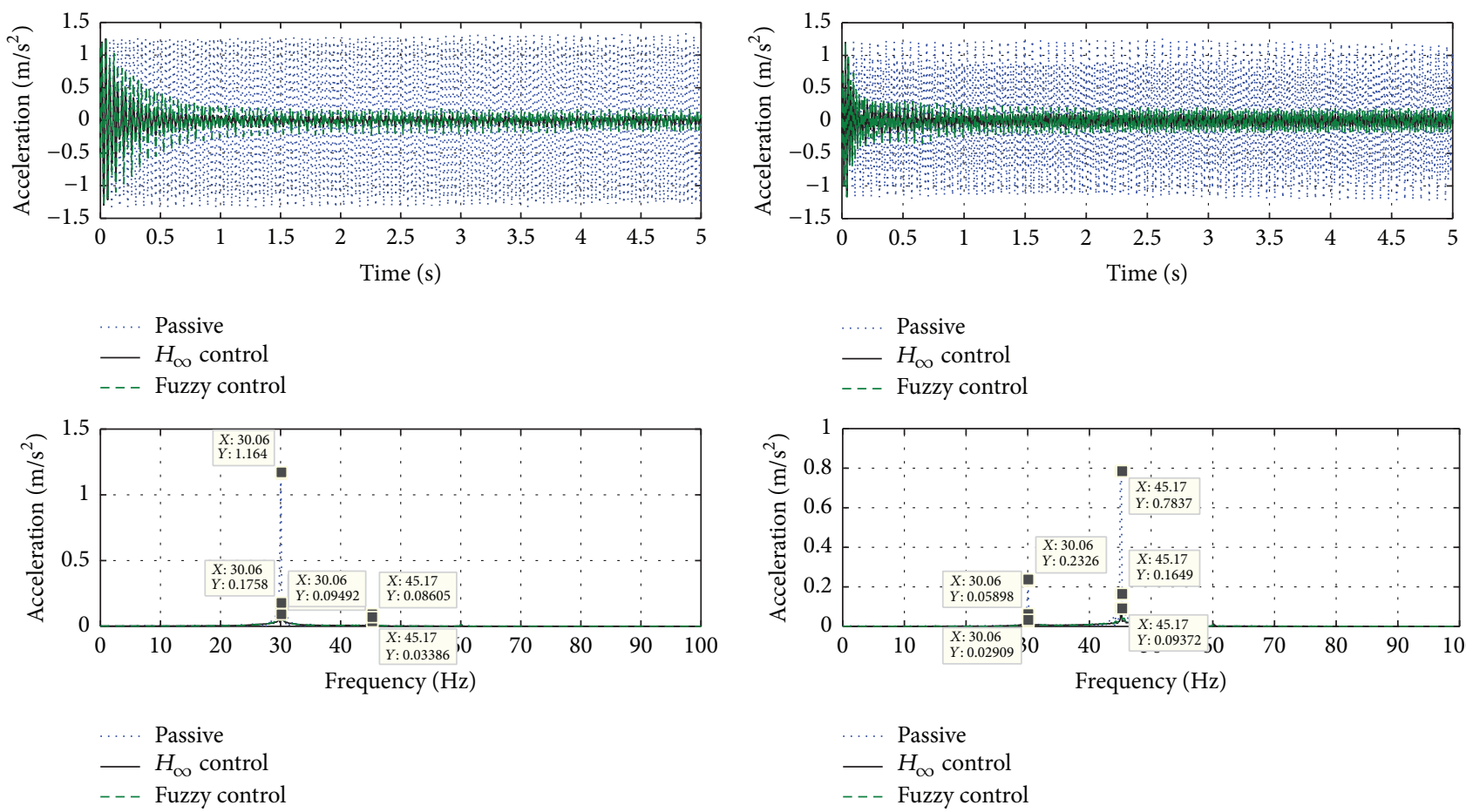

(a)

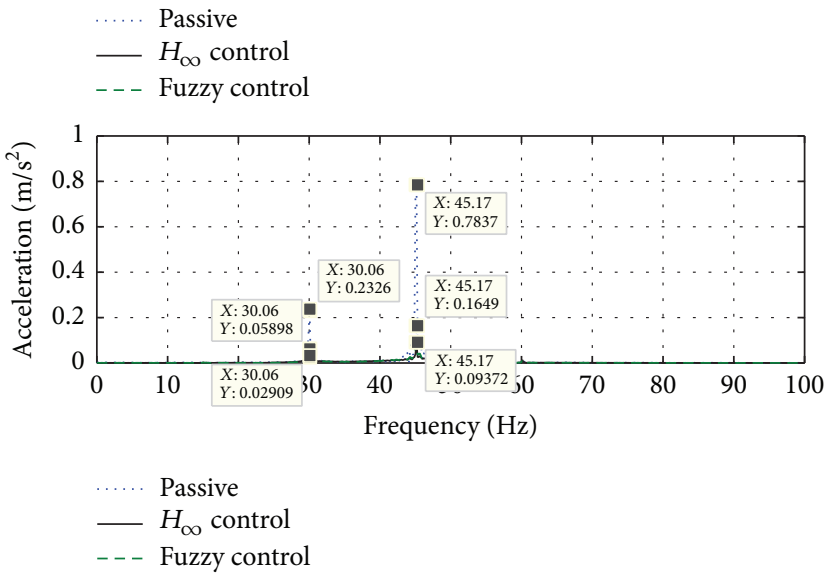

(b)

FiguRE 17: The time-frequency experimental comparison charts of error signals between passive, fuzzy control, and $H_{\infty}$ control.

direction $(Z)$ is the only one of interest in this paper. The upper base and the motors are the vibration source, which are distributed along the center line of the structure and provide a vertical excitation to the upper base floor. The control scheme is shown in Figure 15, too.

In this paper, in order to verify the validity of designed proposal, the identified reduced model from OKID and $H_{\infty}$ controller are applied to the control system of floating raft platform. The operating frequencies of two motors are $30 \mathrm{~Hz}$ and $45 \mathrm{~Hz}$, respectively, which are adjusted by two inverters. The error signals are measured by the accelerometers on the bottom of raft in correspondence of the electromagnetic actuators. The error signals are processed to the suitable amplitude and frequency by the charge amplifier and antialiasing low-pass filter $(1-200 \mathrm{~Hz})$. The dSPACE1103 controller is the real-time system and has seamless connection with MATALB/SIMULINK/RTW. $H_{\infty}$ algorithm is implemented in Simulink and the control algorithm block is proposed in Figure 17. The controller dSAPCE1103 is the real-time system and has seamless connection with Simulink module. $H_{\infty}$ control algorithm code is generated from the Simulink control block by the dSAPCE1103 controller. It is very convenient that the parameters of control block can be adjusted in realtime through the control desk software.

In Figure 16, the error signals $e(k)$ are measured as the input to the dSPACE controller; the output signals $u(k)$ are calculated by $H_{\infty}$ controller, respectively. The control signals $u(k)$ go through the control channels $G(s)$ (including electromagnetic actuator and power amplifier) and then generate the desired output signals $y(k)$. The error signal $e$ is produced by the superposition of the disturbance signal $d$ and the desired output signal $y$. Then $e$ is measured by the sensor and transmitted to the controller $K(s)$. In order to protect the security of the control channel, the output signal voltage is restricted 
between $-5 \mathrm{~V}$ and $+5 \mathrm{~V}$. The ode 8 (Dormand-Prince) is selected as fixed-step solver and fixed-step size is 0.0001 .

Under the same excitation conditions, $H_{\infty}$ control and fuzzy control are executed to reduce the vibration of floating raft platform. Figure 17 presented the time-frequency experimental comparison charts of error signals between passive, fuzzy control, and $H_{\infty}$ control. The amplitude of $1 \#$ error signal declines from $1.2 \mathrm{~m} / \mathrm{s}^{2}$ to $0.2 \mathrm{~m} / \mathrm{s}^{2}$ and becomes stable after $1 \mathrm{~s}$ when fuzzy controller is turned on. For $H_{\infty}$ control, the amplitude is gradually decreased from $1.2 \mathrm{~m} / \mathrm{s}^{2}$ to $0.1 \mathrm{~m} / \mathrm{s}^{2}$. Moreover, the amplitude of $2 \#$ error signal declines from $1.2 \mathrm{~m} / \mathrm{s}^{2}$ to $0.3 \mathrm{~m} / \mathrm{s}^{2}$ and becomes stable after $1 \mathrm{~s}$ when fuzzy controller is turned on. However, the amplitude is still decreased from $1.2 \mathrm{~m} / \mathrm{s}^{2}$ to $0.1 \mathrm{~m} / \mathrm{s}^{2}$ when $H_{\infty}$ control is turned on. More specifically, in the frequency domain diagram, the amplitude of $1 \#$ error signal for $H_{\infty}$ controller and fuzzy control at $30.06 \mathrm{~Hz}$ decreases, respectively, from $1.164 \mathrm{~m} / \mathrm{s}^{2}$ to $0.09492 \mathrm{~m} / \mathrm{s}^{2}$ and $0.1758 \mathrm{~m} / \mathrm{s}^{2}$, which have, respectively, the improvement up to $21.7 \mathrm{~dB}$ and $16.4 \mathrm{~dB}$ compared to the passive system. For the main component of the $1 \#$ error signal is at $45.17 \mathrm{~Hz}, H_{\infty}$ controller and fuzzy control have an improvement which was up to $8.1 \mathrm{~dB}$ and $2.1 \mathrm{~dB}$ when compared to the passive system. The experimental results prove that designed $H_{\infty}$ controller for the multifrequency varying vibration is effective for the floating raft system.

\section{Conclusion}

This paper presents a new approach to state-space model identification of floating raft system from data corrupted by noise. It is based on two main steps: first, estimation of the output residuals of the optimal linear time-invariant observer for the system and the unknown noise statistics, and second, identification of the Kalman filter by solving a new, simpler identification problem. The key feature of the latter is that the newly converted problem is virtually deterministic, which makes any deterministic system identification algorithm for state-space models suitable for its solution. The first step of the method is the same as the first part of the well-known OKID/ERA algorithm. This leads to a generalization of the OKID approach where ERA is not the only algorithm that can complete the identification process as in the original OKID/ERA algorithm. In the new viewpoint, OKID converts the original stochastic system problem into a deterministic one. With this preprocessing step, existing deterministic system identification algorithms can achieve the same performance as traditional stochastic system identification algorithms such as OKID/ERA and OKID/ERA-DC. Examples using experimental data show how the performance of the new algorithms is indeed very similar to these established algorithms. $H_{\infty}$ controller with high robustness to model error is designed to solve the problem of multifrequency varying vibration for floating raft system. Both simulated and experimental results confirm that $H_{\infty}$ control algorithm is an effective approach and has the better performance of reducing multifrequency varying vibration than fuzzy control algorithm for floating raft system.

\section{Competing Interests}

The authors declare that there is no conflict of interests regarding the publication of this paper.

\section{Acknowledgments}

This paper is supported by the National Natural Science Foundation of China (Projects nos. 51275368 and 51205296).

\section{References}

[1] P. Gardonio, S. J. Elliott, and R. J. Pinnington, "Active isolation of structural vibration on a multiple-degree-of-freedom system. Part I: the dynamics of the system," Journal of Sound and Vibration, vol. 207, no. 1, pp. 61-93, 1997.

[2] T. Y. Li, X. M. Zhang, Y. T. Zuo, and M. B. Xu, "Structural power flow analysis for a floating raft isolation system consisting of constrained damped beams," Journal of Sound and Vibration, vol. 202, no. 1, pp. 47-54, 1997.

[3] S. Daley, F. A. Johnson, J. B. Pearson, and R. Dixon, "Active vibration control for marine applications," Control Engineering Practice, vol. 12, no. 4, pp. 465-474, 2004.

[4] J.-S. Bae, J.-H. Hwang, J.-H. Roh, and M.-S. Yi, "Development of an electromagnetic shock absorber," International Journal of Applied Electromagnetics and Mechanics, vol. 49, no. 1, pp. 157167, 2015.

[5] Y. G. Sun, K. J. Song, Y. H. Mao, and L. Lin, "Dynamic analysis of an active flexible suspension system," Journal of Sound and Vibration, vol. 249, no. 3, pp. 606-610, 2002.

[6] D. B. Li and L. H. Yam, "Modal synthesis method for vibration isolation design of massive rotating machines resiliently supported by an elastic structure," Journal of Sound and Vibration, vol. 231, no. 1, pp. 233-245, 2000.

[7] J. C. Snowdon, "Mechanical four-pole parameters and their application," Journal of Sound and Vibration, vol. 15, no. 3, pp. 307-323, 1971.

[8] D. Langhe and K. Sas, "Numerical simulation of waves in beams by using the finite element method," in Proceedings of ISMA19: Tools for Noise and Vibration Analysis, vol. 11, pp. 471-480, Katholieke Universiteit Leuven, 1994.

[9] R. H. Lyon, Statistical Energy Analysis of Dynamical Systems: Theory and Applications, MIT Press, Cambridge, Mass, USA, 1975.

[10] D. Vasic and F. Costa, "Modeling of piezoelectric energy harvester with multi-mode dynamic magnifier with matrix representation," International Journal of Applied Electromagnetics and Mechanics, vol. 43, no. 3, pp. 237-255, 2013.

[11] J. N. Juang, Applied System Identification, Prentice Hall, Englewood Cliffs, NJ, USA, 1994.

[12] J.-N. Juang, M. Phan, L. G. Horta, and R. W. Longman, "Identification of observer/Kalman filter Markov parameters. Theory and experiments," Journal of Guidance, Control, and Dynamics, vol. 16, no. 2, pp. 320-329, 1993.

[13] J.-N. Juang and R. S. Pappa, "An eigensystem realization algorithm for modal parameter identification and model reduction," Journal of Guidance, Control, and Dynamics, vol. 8, no. 5, pp. 620-627, 1985.

[14] J.-N. Juang, J. E. Cooper, and J. R. Wright, "An eigensystem realization algorithm using data correlations (ERA/DC) for modal parameter identification," Control Theory and Advanced Technology, vol. 4, no. 1, pp. 5-14, 1988. 
[15] J. M. Aitken and T. Clarke, "Observer/Kalman filter identification with wavelets," IEEE Transactions on Signal Processing, vol. 60, no. 7, pp. 3476-3485, 2012.

[16] A. Tiano, R. Sutton, A. Lozowicki, and W. Naeem, "Observer Kalman filter identification of an autonomous underwater vehicle," Control Engineering Practice, vol. 15, no. 6, pp. 727-739, 2007.

[17] M. Majji, J.-N. Juang, and J. L. Junkins, "Observer/Kalman-filter time-varying system identification," Journal of Guidance, Control, and Dynamics, vol. 33, no. 3, pp. 887-900, 2010.

[18] T. Clarke and X. D. Sun, "Minimal state-space model realisation of a nonlinear helicopter," IEE Proceedings-Control Theory and Applications, vol. 145, no. 4, pp. 415-422, 1998.

[19] F. Vicario, M. Q. Phan, R. Betti, and R. W. Longman, "Outputonly observer/Kalman filter identification ( $\left.\mathrm{O}^{3} \mathrm{KID}\right)$," Structural Control and Health Monitoring, vol. 22, no. 5, pp. 847-872, 2015.

[20] Q. Xia and T. Shi, "Constraints of distance from boundary to skeleton: for the control of length scale in level set based structural topology optimization," Computer Methods in Applied Mechanics and Engineering, vol. 295, pp. 525-542, 2015.

[21] S. M. Kuo and D. R. Morgan, Active Noise Control Systems: Algorithms and DSP Implementations, John Wiley \& Sons, New York, NY, USA, 1996.

[22] B. Huang, Y. Xiao, J. Sun, and G. Wei, "A variable step-size FXLMS algorithm for narrowband active noise control," IEEE Transactions on Audio, Speech and Language Processing, vol. 21, no. 2, pp. 301-312, 2013.

[23] B. Krstajic, Z. Zecevic, and Z. Uskokovic, "Increasing convergence speed of FxLMS algorithm in white noise environment," $A E U$ - International Journal of Electronics and Communications, vol. 67, no. 10, pp. 848-853, 2013.

[24] M. Tahir Akhtar and W. Mitsuhashi, "Improving performance of FxLMS algorithm for active noise control of impulsive noise," Journal of Sound and Vibration, vol. 327, no. 3-5, pp. 647-656, 2009.

[25] F. Duarte, P. Ballesteros, and X. Shu, "An LPV discrete-time controller for the rejection of harmonic time-fluctuating disturbances in a lightweight flexible structure," in Proceedings of the American Control Conference (ACC '13), pp. 4092-4097, June 2013.

[26] A. J. Connolly, M. Green, and J. F. Chicharo, "The design of LQG and $H_{\infty}$ controllers for use in active vibration control and narrow band disturbance rejection," in Proceedings of the 34th IEEE Conference on Decision and Control, pp. 2982-2987, December 1995.

[27] J. Orivuori, I. Zazas, and S. Daley, "Active control of frequency varying disturbances in a diesel engine," Control Engineering Practice, vol. 20, no. 11, pp. 1206-1219, 2012.

[28] J. C. Doyle, K. Glover, and P. Khargonekar, "State-space solutions to standard $\mathrm{H}_{2}$ and $\mathrm{H}_{\infty}$ control problems," IEEE Transactions on Automatic Control, vol. 8, pp. 831-847, 1989.

[29] A. Karimi and Z. Emedi, " $H_{\infty}$ gain-scheduled controller design for rejection of time-varying narrow-band disturbances applied to a benchmark problem," European Journal of Control, vol. 19, no. 4, pp. 279-288, 2013.

[30] M. Phan, L. G. Horta, J.-N. Juang, and R. W. Longman, "Linear system identification via an asymptotically stable observer," Journal of Optimization Theory and Applications, vol. 79, no. 1, pp. 59-86, 1993.

[31] C. S. Song, Study on basic theory and control technology of magnetic suspension active vibration isolation for flexible floating raft system [Ph.D. thesis], Wuhan University of Technology, 2011.
[32] S. J. Qin and L. Ljung, "Closed-loop subspace identification with innovation estimation," in Proceedings of the 13th IFAC Symposium on System Identification, Rotterdam, The Netherlands, 2003.

[33] W. Lin, S. J. Qin, and L. Ljung, "On consistency of closed-loop subspace identification with innovation estimation," in Proceedings of the 43rd IEEE Conference on Decision and Control (CDC '04), The Bahamas, The Caribbean, December 2004.

[34] F. Vicario, OKID as a general approach to linear and bilinear system identification [Ph.D. thesis], Columbia University, 2014.

[35] C. Song, Z. Zhou, S. Xie, Y. Hu, J. Zhang, and H. Wu, "Fuzzy control of a semi-active multiple degree-of-freedom vibration isolation system," Journal of Vibration and Control, vol. 21, no. 8, pp. 1608-1621, 2015. 


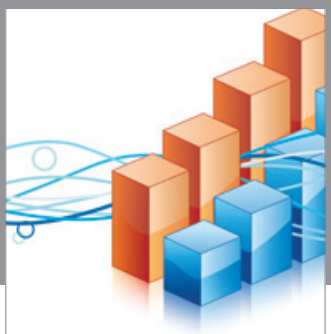

Advances in

Operations Research

vatem alat4

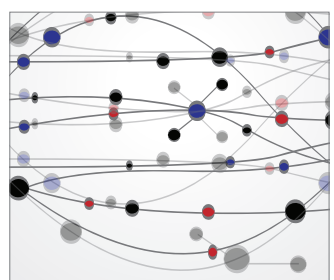

\section{The Scientific} World Journal
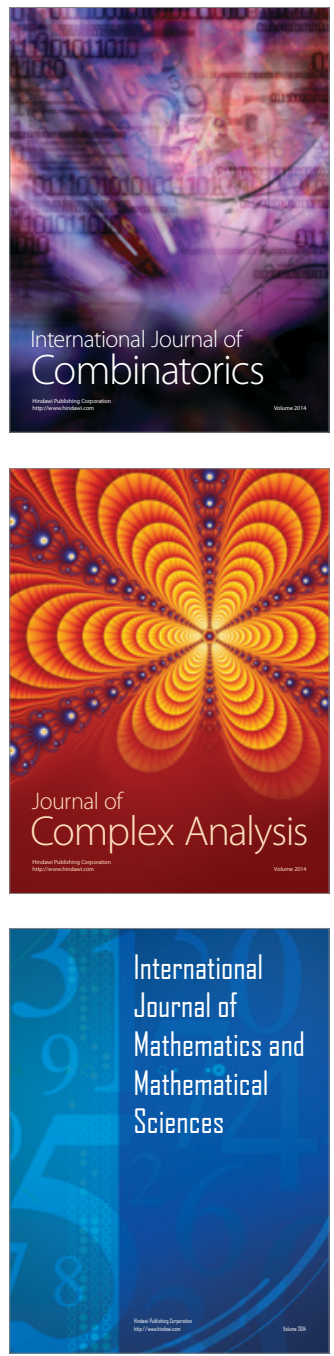
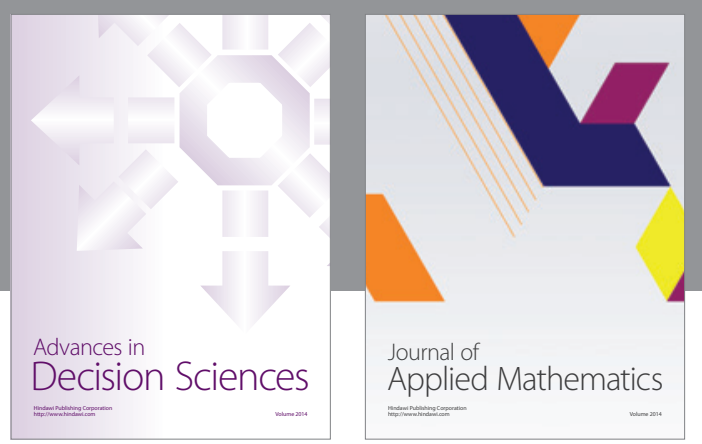

Algebra

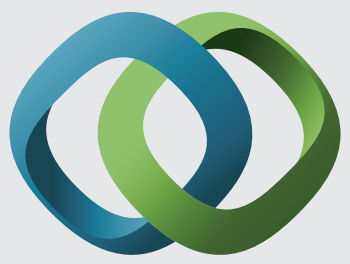

\section{Hindawi}

Submit your manuscripts at

https://www.hindawi.com
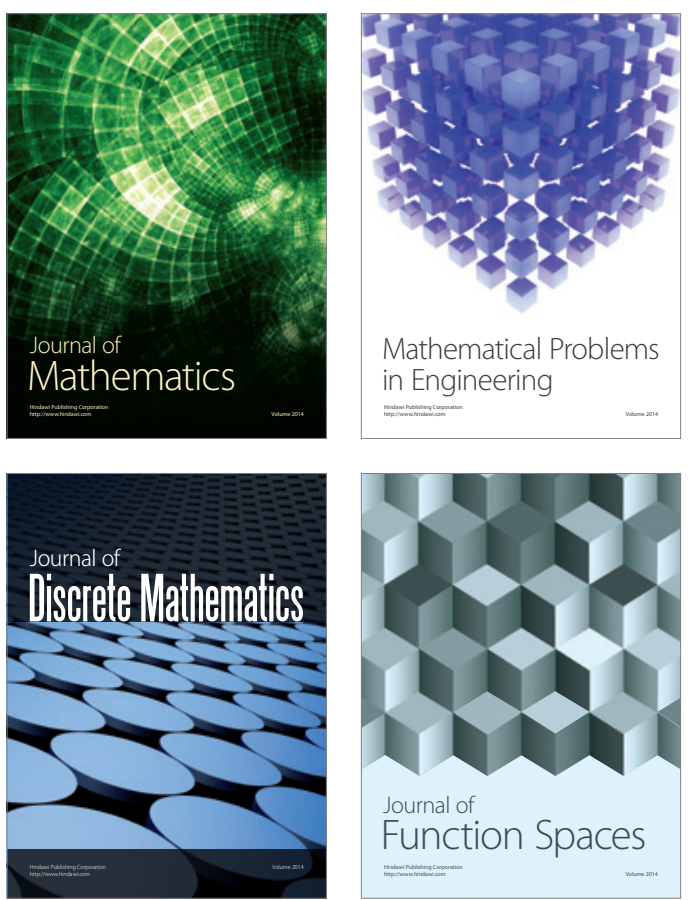

Mathematical Problems in Engineering
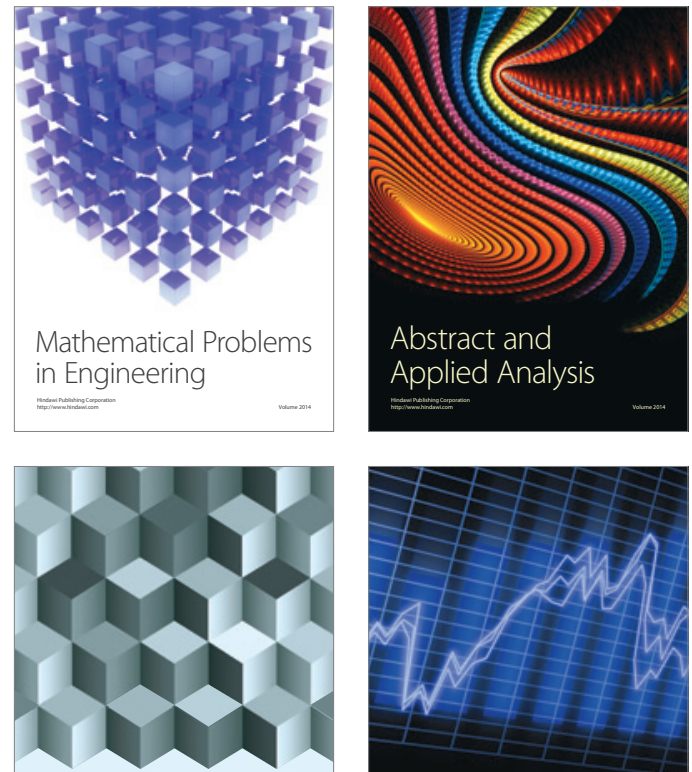

Journal of

Function Spaces

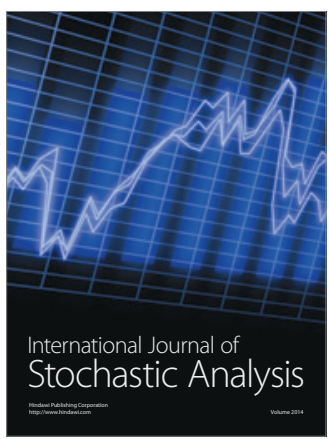

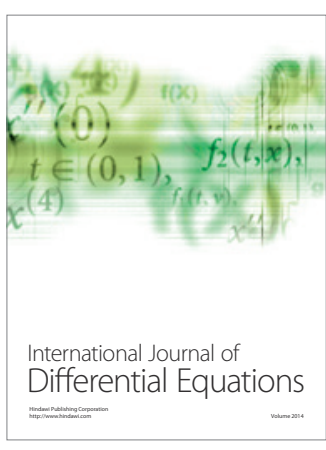
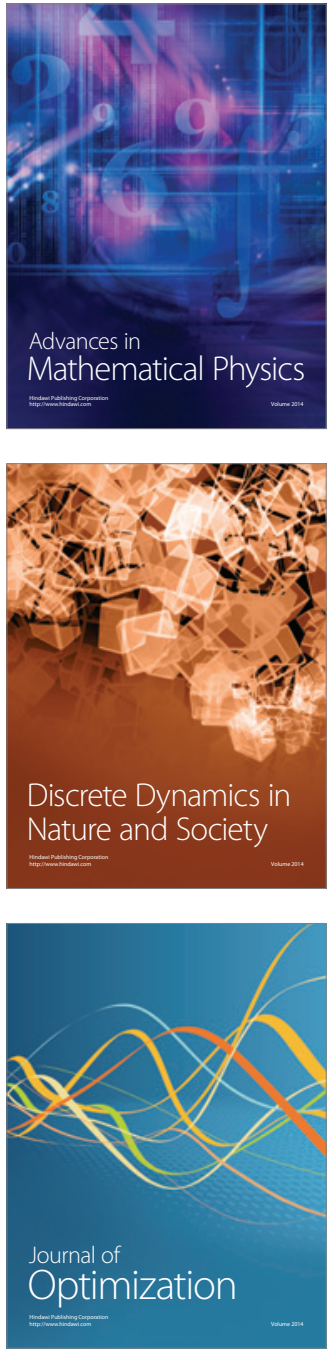